\title{
Article \\ Classification of Flow Modes for Natural Convection in a Square Enclosure with an Eccentric Circular Cylinder
}

\author{
Hyun-Sik Yoon * and Yoo-Jeong Shim
}

check for

updates

Citation: Yoon, H.-S.; Shim, Y.-J.

Classification of Flow Modes for Natural Convection in a Square Enclosure with an Eccentric Circular Cylinder. Energies 2021, 14, 2788. https://doi.org/10.3390/en14102788

Academic Editor: Andrej Kitanovski

Received: 20 April 2021

Accepted: 11 May 2021

Published: 12 May 2021

Publisher's Note: MDPI stays neutral with regard to jurisdictional claims in published maps and institutional affiliations.

Copyright: (c) 2021 by the authors. Licensee MDPI, Basel, Switzerland. This article is an open access article distributed under the terms and conditions of the Creative Commons Attribution (CC BY) license (https:/ / creativecommons.org/licenses/by/ $4.0 /)$.
Department of Naval Architecture and Ocean Engineering, Pusan National University, 2 Busandaehak-ro 63beon-gil, Gumjeong-gu, Busan 46241, Korea; dbwjdking@pusan.ac.kr

* Correspondence: lesmodel@pusan.ac.kr

Abstract: The present study investigated the natural convection for a hot circular cylinder embedded in a cold square enclosure. The numerical simulations are performed to solve a two-dimensional steady natural convection for three Rayleigh numbers of $10^{3}, 10^{4}$ and $10^{5}$ at a fixed Prandtl number of 0.7. This study considered the wide range of the inner cylinder positions to identify the eccentric effect of the cylinder on flow and thermal structures. The present study classifies the flow structures according to the cylinder position. Finally, the present study provides the map for the flow structures at each Rayleigh number $(R a)$. The $R a=10^{3}$ and $10^{4}$ form the four modes of the flow structures. These modes are classified by mainly the large circulation and inner vortices. When $R a=10^{5}$, one mode that existed at $R a=10^{3}$ and $10^{4}$, disappears in the map of the flow structures. The new three modes appear, resulting in total six modes of flow structures at $R a=10^{5}$. New modes at $R a=10^{5}$ are characterized by the top side secondary vortices. The corresponding isotherms are presented to explain the bifurcation of the flow structure.

Keywords: map of flow structures; inner cylinder; enclosure; natural convection

\section{Introduction}

The natural convection for internal bodies embedded in an enclosure has, for many years, been studied in detail because of their fundamental significance in heat transfer physics, and its practical importance in many applications such as heat exchangers, electronic equipment cooling, nuclear and chemical reactors, ventilation or passive building cooling, insulating materials, and food processing. The combination of the conditions of the enclosure and the internal bodies provides various reach topics. Thus, numerous studies have dealt with the natural convection for the internal bodies in the enclosure by considering the individual condition or combined conditions such as the thermal boundary [1-5], the shape [3,4,6-9], 3D effect [2,10-12], the size [8,9,11,13-22], the number of the internal bodies [23], the concentric and eccentric internal body [12,23-40], Rayleigh number [1-26,28,29,31-36,38,39], Prandtl number [31,40,41], the inclined enclosure [18,22], etc. Recently, comprehensive review on this subject was conducted by Pandey et al. [42]. Also, the research for the heat transfer in the enclosure extends to the nanofluid [43-47]. Among the various combinations, the present study considers the 2D cold enclosure with an internal hot circular cylinder. Thus, the natural convection for an internal cylinder embedded in the enclosure is assumed as the relevant topic.

Shu and Zhu [19] numerically investigated the natural convection in a concentric annulus between a heated inner cylinder and a cold square enclosure. They found that the patterns of flow and thermal fields is significantly affected by the aspect ratio and the Rayleigh number $(R a)$.

Kim et al. [26] considered the variation of the vertical position of the inner hot cylinder in $10^{3} \leq R a \leq 10^{6}$. They reported that the dependence of the inner vortices and the primary circulation on the vertical position. Later, Yoon et al. [27] extended the re- 
search of Kim et al. [26] by considering the higher $R a$ of $10^{7}$, showing the occurrence of time dependence.

Hussain and Hussein [28] considered a uniform heat flux as a boundary condition of the inner cylinder alike the problem with Kim et al. [26]. They reported that the effect of a uniform heat flux condition on the Nusselt number in comparison with Kim et al. [26].

Lee et al. [36] considered the change of the cylinder location along the diagonal and horizontal directions in $10^{3} \leq R a \leq 10^{6}$. They revealed that the variation of the mean and local Nusselt numbers accompanying with the modification of the inner cells and the primary circulation according to the cylinder position.

As reviewed above, the natural convection for the internal cylinder in the cavity has been well established by many researches, resulting in greatly improving the understanding of this heat transfer, but based on the authors' literature survey, the previous studies considered the limited positions of the cylinder for the eccentric effect on the natural convection in the enclosure. Mainly three different directions of the vertical, horizontal, and diagonal direction for the position of the cylinder are dealt with the investigation of the eccentric effect.

Therefore, the main emphasis of the present study is to cover the wide range of the internal cylinder positions to identify the eccentric effect on the thermal and flow structures in the enclosure. The present study classifies the flow structures in the enclosure for the inner cylinder location. Finally, the present study provides the maps for the flow structures to reinforce the understanding of the eccentricity of the cylinder within the enclosure for three Rayleigh numbers. The isotherms are presented to explain the bifurcation of the flow structure. The distributions of the Nusselt numbers for the enclosure are presented as the contours in the polar plane embedded in the enclosure in order to match with the map of the flow structures according to the positions of the inner cylinder center.

\section{Numerical Details}

Figure 1 presents a schematic of the present system with the side length $(L)$ of the square enclosure and the cylinder radius of $R=0.2 L$. In order to identify the inner cylinder location, the polar coordinates $(r, \theta)$ are introduced within the enclosure.

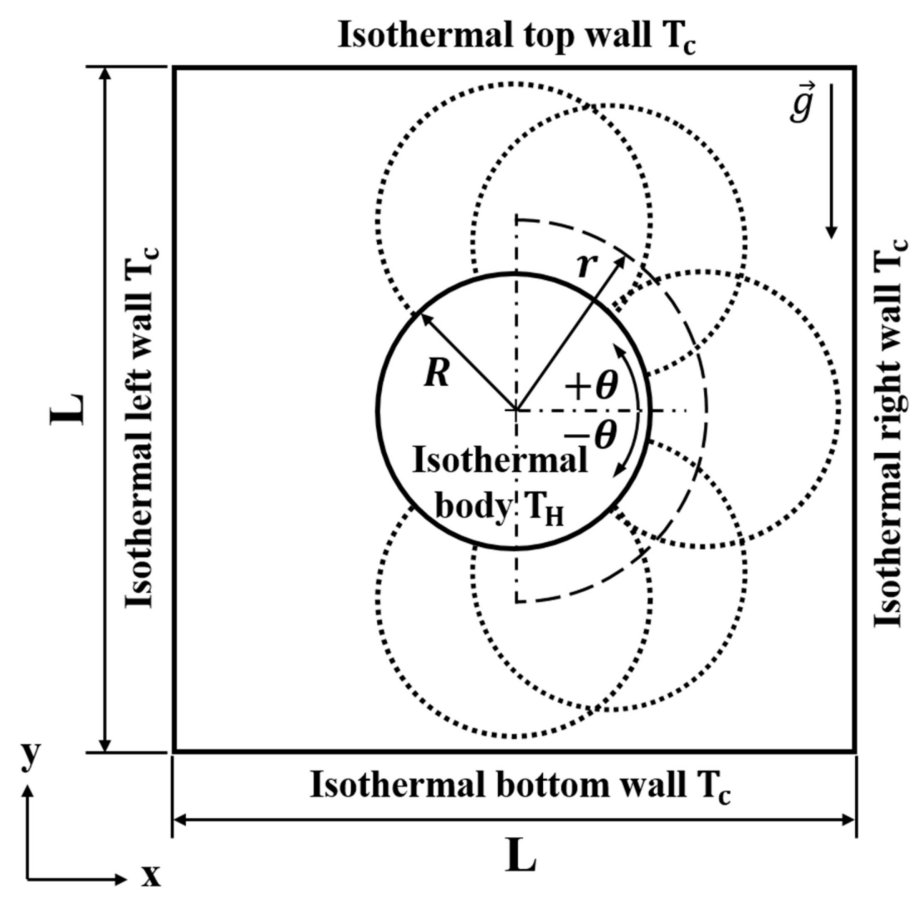

Figure 1. Computational domain and coordinate system along with boundary conditions. 
The origin of the polar coordinate system is the enclosure center. The radial and circumferential positions of the center of the inner cylinder are in the range of $0 \leq r \leq 0.25 \mathrm{~L}$ with intervals of $\Delta r=0.05 \mathrm{~L}$ and $-90^{\circ} \leq \theta \leq 90^{\circ}$ with intervals of $\Delta \theta=10^{\circ}$, respectively. To resolve the diagonal positions, $\theta=45^{\circ}$ and $\theta=-45^{\circ}$ are also considered. The solutions of the present problems are steady and symmetric about $x=0$. Thus, we consider the cylinder positions in the right half of the enclosure. In total $10^{6}$ positions of the inner cylinder are considered in this study.

The constant low temperature $\left(T_{c}\right)$ and high temperature $\left(T_{h}\right)$ are put on the enclosure walls and the cylinder surface, respectively. The present study does not consider the radiation which is assumed to be negligible. The Boussinesq approximation is used for the density. The constant assumption is used for the rest of properties. Thus, under the present assumptions, the following continuity, momentum and energy equations satisfying the unsteady incompressible viscous flow and the thermal fields are the dimensionless governing equations:

$$
\begin{gathered}
\frac{\partial u_{i}^{*}}{\partial x_{i}^{*}}=0 \\
\frac{\partial u_{i}^{*}}{\partial t^{*}}+u_{j}^{*} \frac{\partial u_{i}^{*}}{\partial x_{j}^{*}}=-\frac{\partial p^{*}}{\partial x_{i}^{*}}+\operatorname{Pr} \frac{\partial^{2} u_{i}^{*}}{\partial x_{j}^{*} x_{j}^{*}}+\operatorname{RaPr} T^{*} \delta_{i 2} \\
\frac{\partial T^{*}}{\partial t}+u_{j}^{*} \frac{\partial T^{*}}{x_{j}^{*}}=\frac{\partial^{2} T^{*}}{\partial x_{j}^{*} \partial x_{j}^{*}}
\end{gathered}
$$

where, the superscript $*$ represents the dimensionless variable. In the above equations, $u_{i}$ are the components of velocity corresponding to the Cartesian coordinates of $x_{i}, p$ is the pressure, $t$ is the time and $T$ is the temperature. The dimensionless variables in the above equations are defined as:

$$
t^{*}=\frac{t \alpha}{L^{2}}, x_{i}^{*}=\frac{x_{i}}{L}, u_{i}^{*}=\frac{u_{i} L}{\alpha}, p^{*}=\frac{p L^{2}}{\rho \alpha^{2}}, T^{*}=\frac{T-T_{c}}{T_{h}-T_{c}}
$$

where, $\alpha$ and $\rho$ denote the thermal diffusivity and the density, respectively.

The non-dimensionalization results in dimensionless parameters of Rayleigh number, $R a=\frac{g \beta L^{3}\left(T_{h}-T_{c}\right)}{v \alpha}$ and Prandtl number, $P r=v / \alpha$, where $\beta, g$ and $v$ are the expansion coefficient, the gravitational acceleration and the kinematic viscosity, respectively. The value of $\operatorname{Pr}$ is 0.7 which corresponds to the air. The present study considers three values of $R a$ of $10^{3}, 10^{4}$ and $10^{5}$.

For the discretization of the space, a second-order central difference scheme is utilized to structured grids, based on the finite volume method (FVM). A second-order implicit scheme is employed for temporal discretization. The Crank-Nicolson scheme and the second-order Adams-Bashforth are adopted for the diffusion and convection terms, respectively. A two-step, time-split scheme is used for the time advance of the flow and thermal fields. The solution of Poisson equation satisfies the continuity equation. Further details of this scheme are can be referred from Kim and Moin [48] and Zang et al. [49]. We derived the governing equations using a generalized curvilinear coordinate system.

The boundary conditions of the no-penetration and no-slip are imposed on the walls for the flow. As the wall boundaries for the energy equation, the hot and cold isothermal conditions are defined for the internal cylinder and the enclosure, respectively.

After achieving the velocity and temperature, the local and surface-averaged Nusselt numbers are obtained by following definitions;

$$
N u=\left.\frac{\partial T^{*}}{\partial n}\right|_{\text {wall }}, \overline{N u}=\frac{1}{A} \int_{0}^{A} N u d S
$$

where $n$ and $A$ are the normal direction to the wall and the surface area. 
Figure 2 shows the present grid system. The present computations use the grid numbers of $201 \times 201$ in the horizontal $(x)$ and vertical $(y)$ directions. The non-uniform grid distribution is generated by using the algebraic function to resolve the high gradients near the walls. Particularly, the dense resolution of the grid is maintained by tuning the grid points to cover the variation of the cylinder position. The test of the grid dependence is performed by using coarser and much finer grids of $102(x) \times 102(y), 303(x) \times 303(y)$ and $402(x) \times 402(y)$. The difference of Nusselt numbers between grid numbers becomes smaller, which is less than $0.5 \%, 0.1 \%$ and $0.07 \%$ for the coarse, medium and fine grids. Finally, the grid resolution of $201(x) \times 201(y)$ is used in all commutations for the computational efficiency.

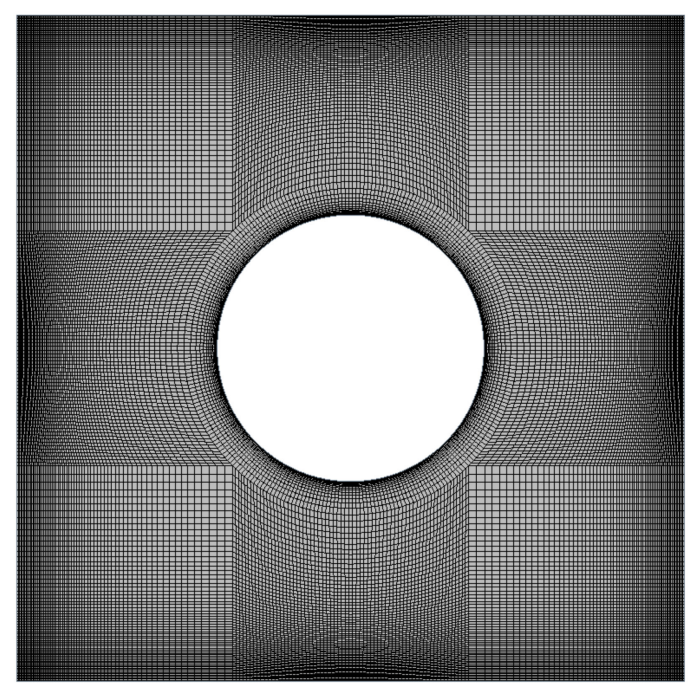

Figure 2. A typical grid distribution for $r=0$.

The convergence criteria is $10^{5}$. The present study adopts the variable time step in order to meet the numerical stability condition of the Courant-Friedrichs-Lewy condition $(C F L), C F L \leq 0.3$, for all calculations.

For the validation of the present numerical methods, the surface-averaged Nusselt numbers of the inner cylinder for different values of $R a$ are compared with those of Park et al. [18], Kim et al. [26] and Lee et al. [36], as presented in Table 1. The present results agree well with the previous researches.

Table 1. Comparison of surface-averaged Nusselt number at hot wall from this study with those of previous numerical studies.

\begin{tabular}{cccccccc}
\hline \multirow{2}{*}{ Ra } & Present & \multicolumn{2}{c}{ Park et al. [18] } & \multicolumn{2}{c}{ Kim et al. [26] } & \multicolumn{2}{c}{ Lee et al. [36] } \\
\cline { 2 - 8 } & $\left\langle\overline{\boldsymbol{N u}}_{c y}\right\rangle$ & $\left\langle\overline{\boldsymbol{N u}}_{c y}\right\rangle$ & Diff. (\%) & $\left\langle\overline{\boldsymbol{N u}}_{c y}\right\rangle$ & Diff. (\%) & $\left\langle\overline{\boldsymbol{N u}}_{c y}\right\rangle$ & Diff. (\%) \\
\hline $10^{3}$ & 5.041 & 5.024 & -0.346 & 5.093 & 1.024 & 5.107 & 1.285 \\
$10^{4}$ & 5.132 & 5.129 & -0.064 & 5.108 & -0.473 & 5.109 & -0.456 \\
$10^{5}$ & 7.816 & 7.817 & 0.010 & 7.767 & -0.630 & 7.761 & -0.712 \\
\hline
\end{tabular}

In addition, in order to identify the reliability of the present numerical methods to achieve the solutions of the eccentricity between the enclosure and the cylinder, the previous results $[26,40]$ for total surface-averaged $N u$ of the square enclosure $\left(\overline{N u}_{e n}\right)$ and the inner circular cylinder $\left(\overline{N u}_{c y}\right)$ for the vertical variation are considered. The present results agree well with the previous results $[26,40]$, as presented in Figure 3. 


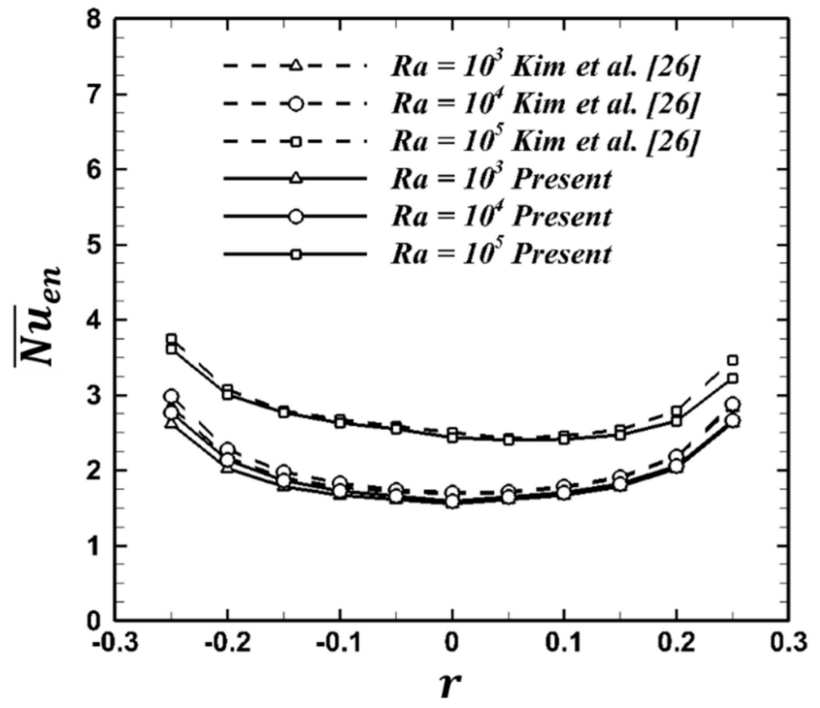

(a)

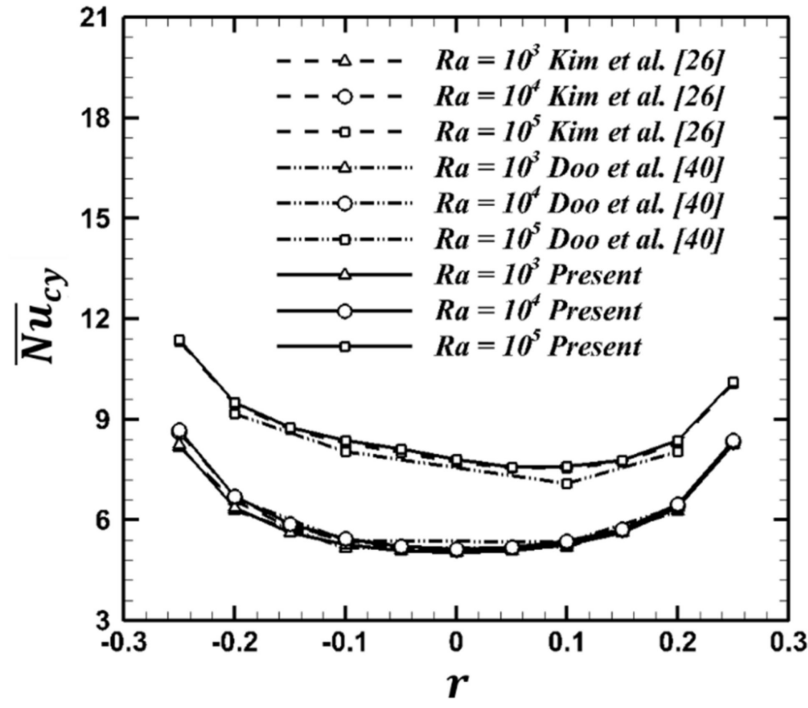

(b)

Figure 3. Comparison of present results of total surface-averaged Nusselt number with Kim et al. [26] and Doo et al. [40]: (a) the square enclosure, $\overline{N u}_{e n}$ and (b) the inner circular cylinder, $\overline{N u}_{c y}$.

\section{Results and Discussion}

\subsection{Map and Patterns of Flow and Thermal Fields}

The map is introduced to show the patterns of thermal and flow fields in the $(r, \theta)$ plane which covers the inner cylinder positions. In order to reinforce the understanding of the eccentricity of the cylinder within the enclosure, the $(r, \theta)$ plane for the map is inserted in the enclosure, as shown in Figure 4.

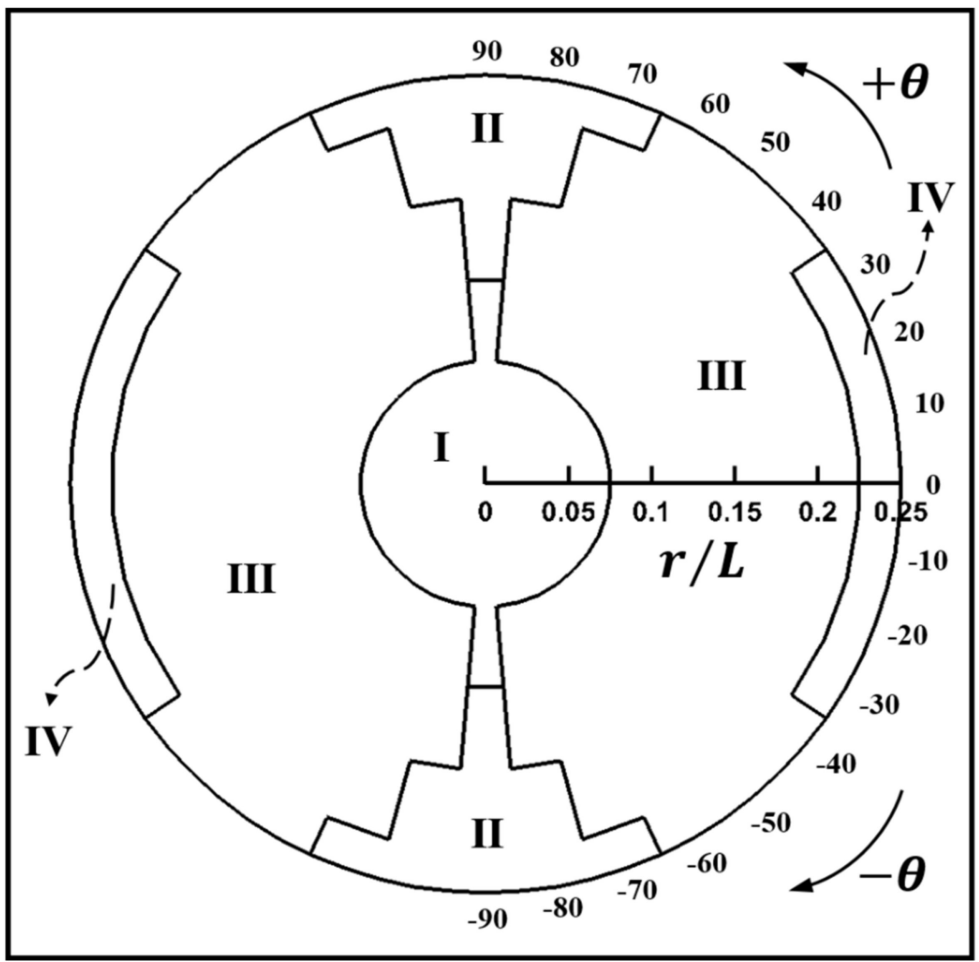

Figure 4. Map of the flow structure for $R a=10^{3}$. 


\subsection{1. $R a=10^{3}$}

Figure 4 show the map of the flow structures for $R a=10^{3}$. The flow structures are classified based on the formation of the streamlines. At low this $R a$, the conduction mode is predominant to the heat transfer in the enclosure. The map of the flow structures for $R a=10^{3}$ is a four-fold symmetry about the horizontal and vertical center lines at $y=0$ and $x=0$, respectively.

In general, the Mode I covers the circular formation with the enclosure center and extends to the upper and lower narrow areas long the vertical center line. At the enclosure center of $r=0$ and $\theta=0^{\circ}$, the isothermal lines are almost linearly distributed along the radial direction. The corresponding streamlines reveal two inner vortices embedded in two symmetric circulations, as presented in Figure 5a. Therefore, Mode I reveals two overall rotating primary eddies and each rotating eddy has two inner vortices (TPE_TIV).

When the internal cylinder positioned below the horizontal center of $y=0$ and moves to lower boundary of Mode I, the area between the bottom wall and the cylinder becomes narrow. Thus, the size of lower inner vortex is reduced gradually, but the upper inner vortex is getting bigger. The corresponding isotherms is getting denser in the region between the bottom wall and the inner cylinder, as shown in Figure $5 \mathrm{~b}$ for $(r=0.1 L$, $\left.\theta=-90^{\circ}\right)$. The thermal and flow fields for $\left(r=0.1 L, \theta=90^{\circ}\right)$ are symmetric with those for $\left(r=0.1 L, \theta=-90^{\circ}\right)$. This variation of each fields in Mode I is consistent with the findings of Kim et al. [26].
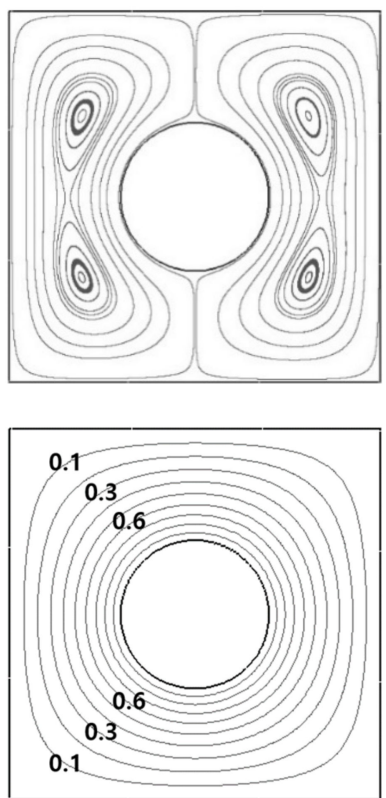

(a) Mode I
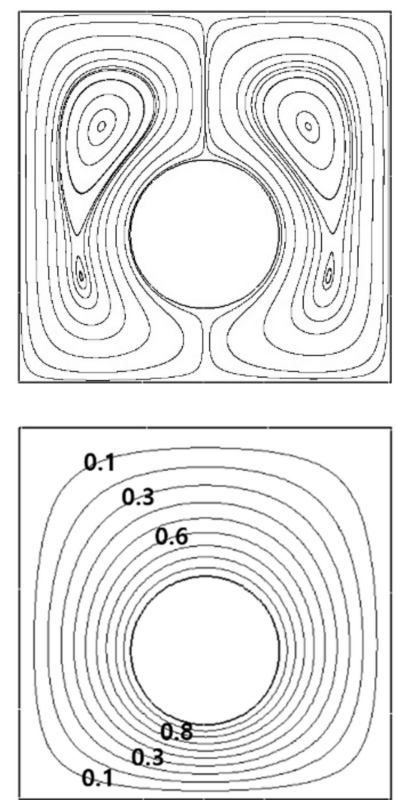

(b) Mode I
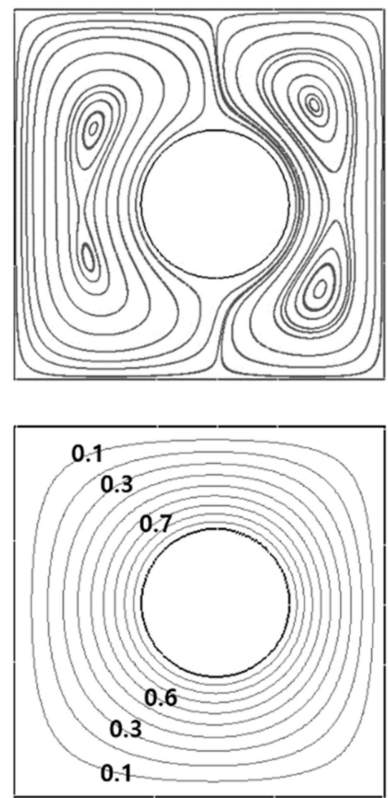

(c) Mode I

Figure 5. Streamlines (upper row) and isotherms (lower row) at (a) $\left(r=0, \theta=0^{\circ}\right),(\mathbf{b})(r=0.1 L$, $\left.\theta=-90^{\circ}\right)$ and $(\mathbf{c})\left(r=0.05 \mathrm{~L}, \theta=30^{\circ}\right)$ for $R a=10^{3}$.

In Mode I, in order to investigate the effect of the circumferential position on the thermal and flow fields, the variation of the circumferential direction is considered from the vertical center line $\left(\theta=90^{\circ}\right.$ and $\left.\theta=-90^{\circ}\right)$ to the horizontal center line $\left(\theta=-0^{\circ}\right)$. When $\theta$ approaches counterclockwise from $\theta=-90^{\circ}$ to $\theta=0^{\circ}$ within Mode I, the two inner vortices in the left and right primary counter-clockwise eddies become smaller and larger, respectively, as shown in Figure $5 \mathrm{c}$ for $\theta=-30^{\circ}$. Consequently, when $\theta$ approaches counterclockwise from $\theta=-90^{\circ}$ to $\theta=0^{\circ}$, the distance between the right wall and inner cylinder enclosure becomes shorter. Thus, the gradient of isotherms augments, as the primary eddy becomes smaller in the right side. When $\theta$ approaches clockwise from $\theta=90^{\circ}$ to $\theta=0^{\circ} \theta=0^{\circ}$ within Mode I, the thermal and flow fields undergo the symmetric 
pattern about the horizontal center line at $y=0$ to those for the counterclockwise rotation from $\theta=-90^{\circ}$ to $\theta=0^{\circ}$.

When the inner cylinder gets away from the boundary of Mode I for $\left(r=0.1 L, \theta=90^{\circ}\right)$ and move downward and upward, two inner vortices are merged into the primary eddy at $\left(r=0.15 \mathrm{~L}, \theta=90^{\circ}\right)$ in Figure $6 \mathrm{a}$ and $\left(r=0.15 \mathrm{~L}, \theta=-90^{\circ}\right)$ which is symmetry to $(r=0.15 \mathrm{~L}$, $\left.\theta=90^{\circ}\right)$. Therefore, Mode II reveals two overall rotating primary eddies (TPE). The area of Mode II become wider in the circumferential direction with increasing the redial position of the inner cylinder center. The critical circumferential locations for the radial positions of $r=0.2 \mathrm{~L}$ and $r=0.25 \mathrm{~L}$ are $\theta=80^{\circ}$ and $\theta=70^{\circ}$, respectively, as shown in Figure 4 for the Map. Thus, as the radial position increases, the critical circumferential location, where the flow structure changes, decreases.
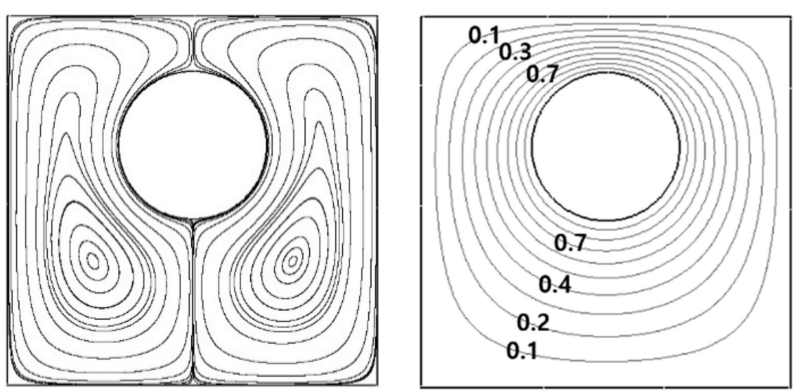

(a) Mode II
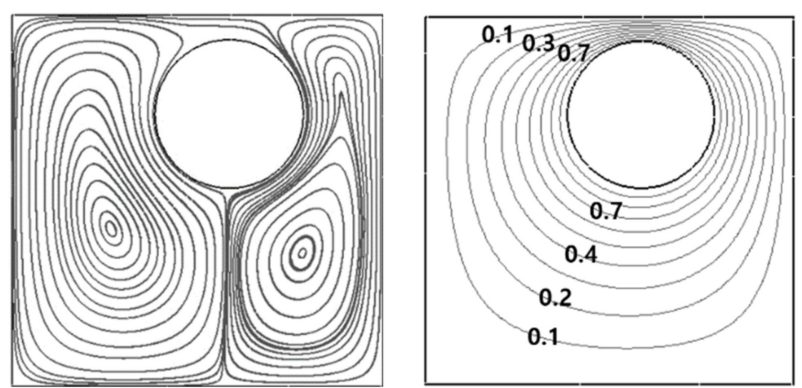

(b) Mode II

Figure 6. Streamlines (left column) and isotherms (right column) at (a) $\left(r=0.15 L, \theta=90^{\circ}\right),(\mathbf{b})\left(r=0.25 L, \theta=70^{\circ}\right)$ for $R a=10^{3}$.

The effect of the cylinder radial position is that the core of the primary circulation moves toward the same direction of the variation of the internal cylinder location because the enclosure can secure spaces opposite the inner cylinder moving enough to enlarge the core of the circulation. When $r$ increases within Mode II, isotherms become denser in between the cylinder and the enclosure wall facing to the cylinder, otherwise isotherms are getting sparser in the opposite region.

In Mode II, the effect of the circumferential position on the flow and thermal fields is almost the same as Mode I. When the inner cylinder rotates clockwise from $\theta=90^{\circ}$ to $\theta=70^{\circ}$ within Mode II, the space in the direction of the inner cylinder rotating becomes small, as shown in Figure 6b. As a result, in this space, the primary circulations are squeezed and small. The isotherms form dense and coarse gradients in the cylinder side and opposite regions, respectively. The counter-clockwise rotation from $\theta=-90^{\circ}$ to $\theta=-70^{\circ}$ within Mode II undergoes the same variation as the clockwise rotation from $\theta=90^{\circ}$ to $\theta=70^{\circ}$.

When the inner cylinder gets away from the boundary of Mode I for $\left(r=0.1 L, \theta=90^{\circ}\right)$ and rotates clockwise from $\theta=90^{\circ}$ to $\theta=80^{\circ}$, in the left side of the enclosure, two secondary inner eddies at $\left(r=0.1 L, \theta=90^{\circ}\right)$ is merged into the primary eddy at $\left(r=0.1 L, \theta=80^{\circ}\right)$, as shown in Figure 7a. When $\theta$ continuously turn clockwise from $\theta=80^{\circ}$ to $\theta=0^{\circ}$, the center of the merged inner vortex in the left circulation rotates clockwise, as shown in Figure 7a,b for $\theta=80^{\circ}$ to $\theta=80^{\circ}$, respectively. Simultaneously, in the right primary eddy, the upper inner vortex becomes bigger and the lower inner vortex squeezes into the clockwise direction.

Therefore, Mode III reveals two overall rotating primary eddies which have two inner vortices in the primary circulation in the direction of the inner cylinder rotation and one inner vortex in the opposite primary circulation (TPE_TOIV). When the inner cylinder gets away from the boundary of Mode II holding TPE, the primary circulation in the inner cylinder rotating direction contains additional inner vortex. When the inner cylinder circumferentially approaches to the horizontal center $\left(\theta=0^{\circ}\right)$, this additional inner vortex becomes bigger and the lower inner vortex squeezes into the clockwise direction. When $\theta$ approaches counter-clockwise from $\theta=-90^{\circ}$ with the negative sign in the lower half of the enclosure to $\theta=0^{\circ}$ within Mode III, the flow and thermal fields undergo the symmetric 
pattern about the horizontal center line to those for the clockwise rotation from $\theta=90^{\circ}$ to $\theta=0^{\circ}$.
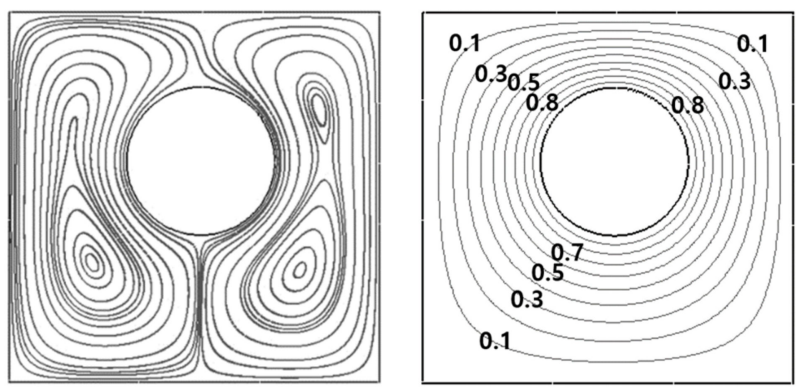

(a) Mode III
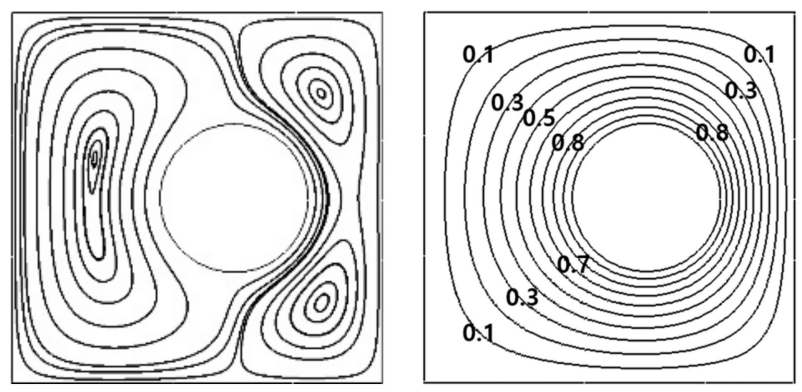

(b) Mode III

Figure 7. Streamlines (left column) and isotherms (right column) at (a) $\theta=80^{\circ}$ and (b) $\theta=0^{\circ}$ at a fixed $r=0.1 \mathrm{~L}$ for $R a=10^{3}$.

When the position of the inner cylinder changes along the radial direction, gets away from the boundary of Mode II and enters into Mode IV, the primary eddy in the direction of the cylinder moving are divided in Figure 8 for $r=0.1 \mathrm{~L}$ and $\theta=0^{\circ}$. Also, when the inner cylinder rotates along the circumferential direction, gets away from the boundary of Mode III and enters into Mode IV, two inner vortices in the primary eddy are divided in the direction of the inner cylinder rotation. Therefore, Mode IV reveals one overall rotating primary eddy and two divided vortices (OPE_TDV).

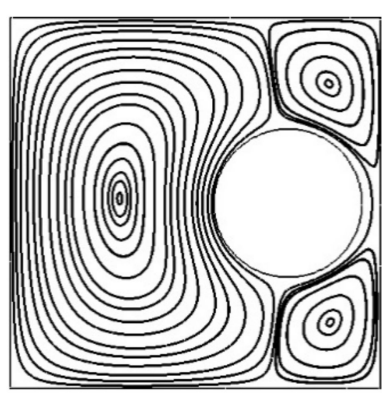

(a)

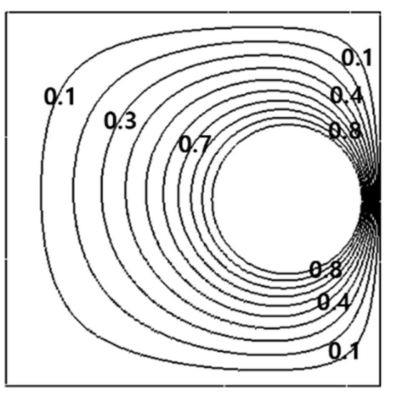

(b)

Figure 8. Streamline (a) and isotherm (b) at $\left(r=0.25 \mathrm{~L}, \theta=0^{\circ}\right)$ in Mode IV for $R a=10^{3}$.

\subsection{2. $R a=10^{4}$}

The isothermal lines and streamlines at $R a=10^{4}$ have almost the same formations as those at $R a=10^{4}$. For each mode, the effects of the radial and circumferential locations of the internal cylinder center on the thermal and flow fields at $R a=10^{4}$ are very similar to those at $R a=10^{4}$. Thus, the map of the flow structures for $R a=10^{4}$ consists with the same four modes of Mode I, Mode II, Mode II and Mode IV corresponding to TPE_TIV, TPE, TPE_TOIV, and OPE_TDV, respectively, as $R a=10^{3}$. However, the buoyance in the direction of the gravity gives a greater effect on both fields, because of the augmentation of the convection. Thus, in contrast to $R a=10^{3}$ having a four-fold symmetry, the map for $R a=10^{4}$ is a two-fold symmetry $x=0$, as presented in Figure 9 .

In general, the $R a=10^{4}$ case forms thinner thermal boundary layer on the cylinder bottom than the upper side, and a smaller and weaker inner lower vortex than the upper one, comparing to $R a=10^{3}$. The regions of Modes I, II and IV for $R a=10^{4}$ are larger than the regions of corresponding Modes for $R a=10^{3}$, as shown in Figures 4 and 9, respectively. 


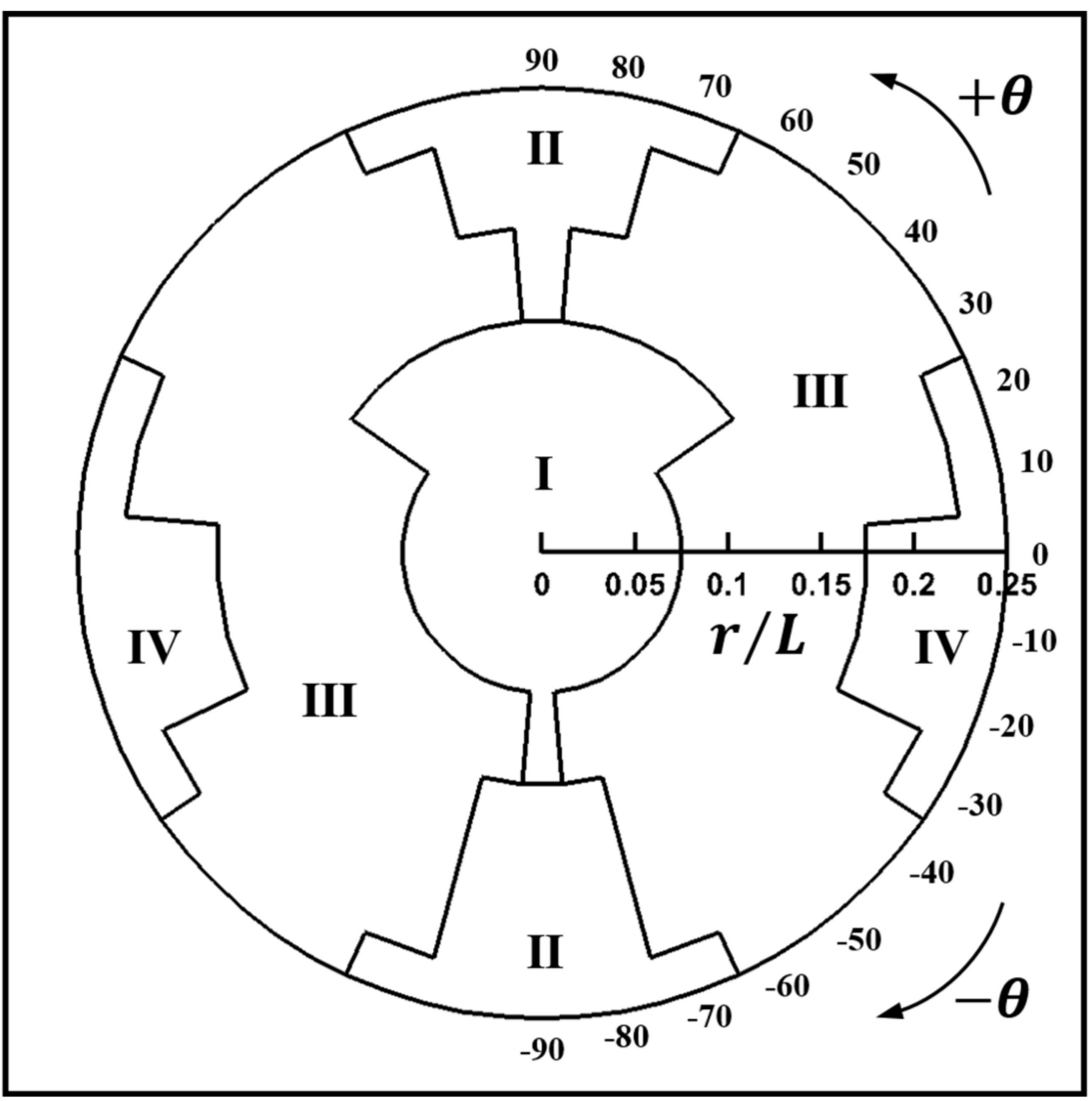

Figure 9. Map of the flow structure for $R a=10^{4}$.
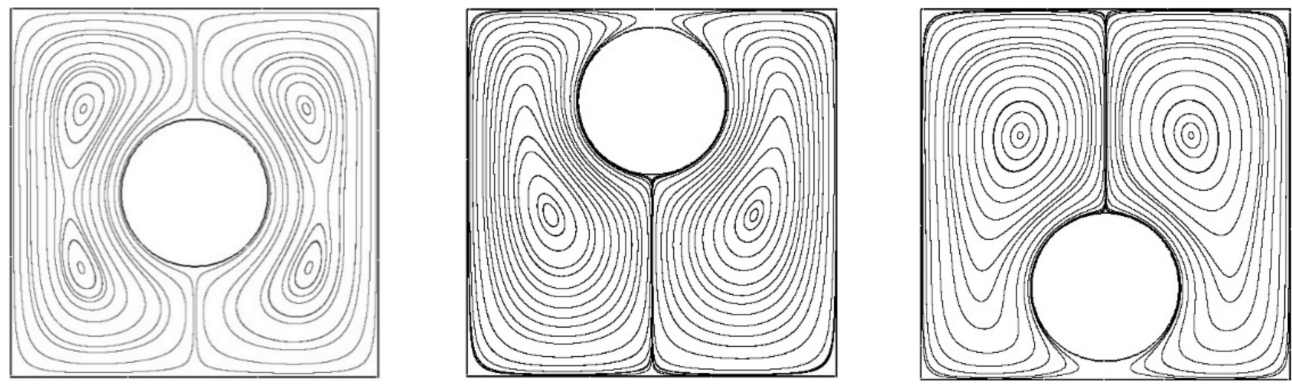

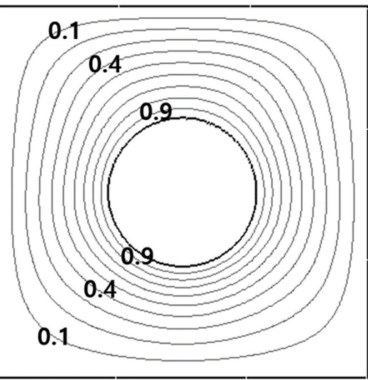

(a) Mode I

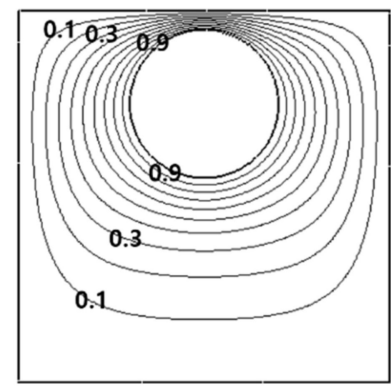

(b) Mode II

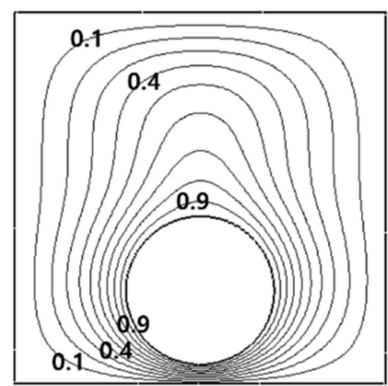

(c) Mode II

Figure 10. Streamlines (upper row) and isotherms (lower row) at (a) $\left(r=0, \theta=0^{\circ}\right),(\mathbf{b})(r=0.25 L$, $\left.\theta=90^{\circ}\right)$ and $(\mathbf{c})\left(r=0.25 \mathrm{~L}, \theta=-90^{\circ}\right)$ for $R a=10^{4}$.

As the cylinder is positioned downward from the enclosure at $r=0$ along the vertical center line at $\theta=-90^{\circ}$, two internal vortices merges at $r=0.1 \mathrm{~L}$. Namely, Mode I changes into Mode II, which is earlier than $r=0.15 \mathrm{~L}$ at $R a=10^{3}$. In Mode II, as $r$ increases at 
$\theta=-90^{\circ}$, isotherms becomes distorted and the thermal plumes on the cylinder top is getting bigger.

As the cylinder is located upward at $\theta=90^{\circ}$, the occurrence of the bifurcation from Mode I to Mode II at $r=0.15 \mathrm{~L}$ is later than that for the downward movement of the cylinder, due to the stronger convective flow in the space between the top wall and the cylinder.

In Mode II formation, when the cylinder is positioned further upward, the two overall rotating primary eddies are more obligated than the downward movement of the cylinder, as observed in Figure 10b,c. These present results for the vertical eccentric effect of the cylinder on the isotherms and streamlines are consistent with Kim et al.'s [26] ones.

The variation of the circumferential position of the cylinder for $R a=10^{4}$ gives a similar effect on the natural convection as $R a=10^{3}$. But, the sizes of the Modes for $R a=10^{4}$ are different with those for $R a=10^{3}$. When the inner cylinder rotates clockwise from $\theta=90^{\circ}$ to $\theta=0^{\circ}$ at $r=0.1 \mathrm{~L}$ in the upper region, the flow structures changes from Mode I to Mode III, as shown in $\theta=90^{\circ}, \theta=30^{\circ}$ and $\theta=0^{\circ}$ in Figure 11a-c, respectively, where the critical angle for the bifurcation of flow structures is $\theta=30^{\circ}$. However, when the inner cylinder rotates counter-clockwise from $\theta=-90^{\circ}$ to $\theta=0^{\circ}$ at $r=0.1 \mathrm{~L}$ in the lower region, the flow structures changes from Mode I to Mode III, as shown in $\theta=-80^{\circ}$ in Figure $11 \mathrm{~d}$, where the critical angle is $\theta=-80^{\circ}$.
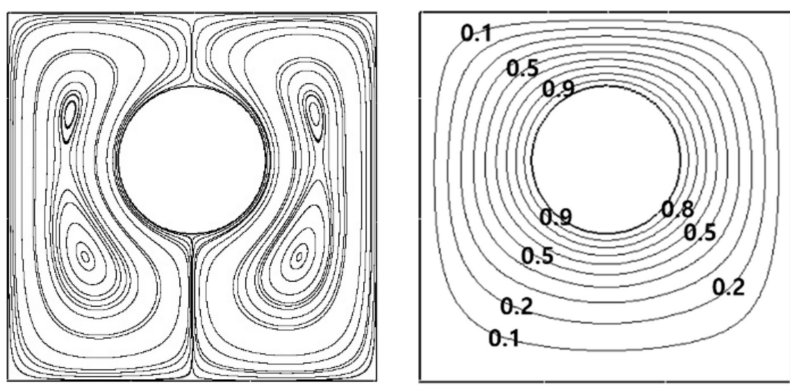

(a) Mode I
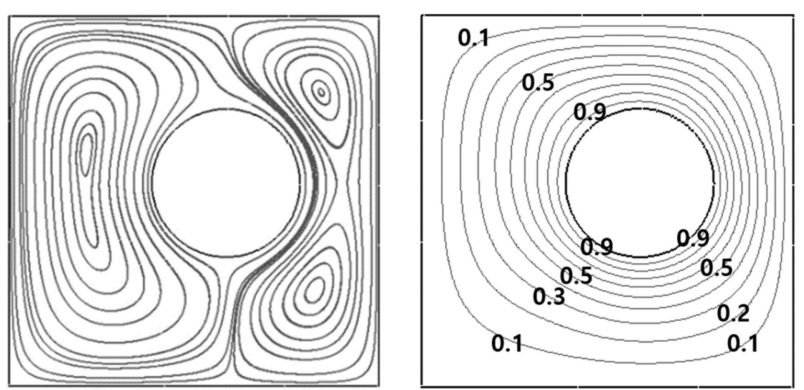

(b) Mode III
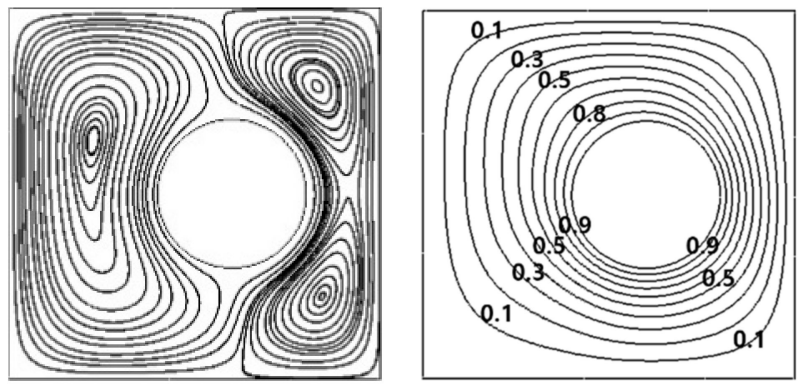

(c) Mode III
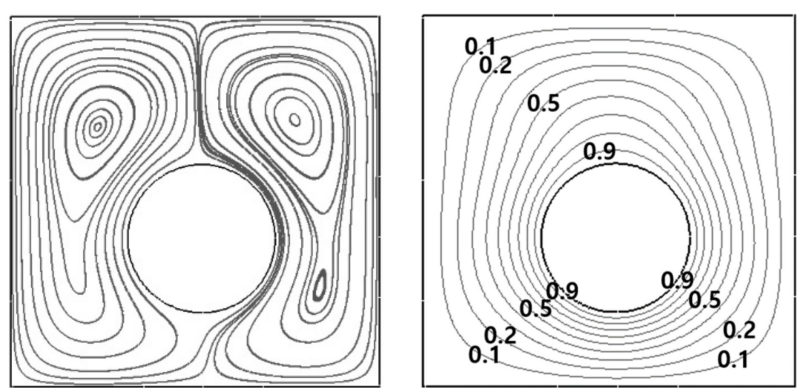

(d) Mode III

Figure 11. Streamlines (left column) and isotherms (right column) at (a) $\theta=90^{\circ}$, (b) $\theta=30^{\circ}$, (c) $\theta=0^{\circ}$ and (d) $\theta=-80^{\circ}$ at a fixed $r=0.1 L$ for $R a=10^{4}$.

At $r=0.2 L$ and $r=0.25 \mathrm{~L}$, the flow structures undergo three modes by the effect of the circumferential eccentricity of the cylinder. At $r=0.2 L$, when the cylinder rotates clockwise from $\theta=90^{\circ}$ to $\theta=0^{\circ}$, Mode II changes into Mode III at $\theta=70^{\circ}$ and then the bifurcation from Mode III to Mode IV occurs at $\theta=0^{\circ}$, as presented in Figure 12a-c, respectively. When the internal cylinder rotates counter-clockwise from $\theta=-90^{\circ}$ to $\theta=0^{\circ}$, Mode II changes into Mode III at $\theta=-70^{\circ}$ and then the bifurcation from Mode III to Mode IV occurs at $\theta=-20^{\circ}$, as presented in Figure $12 \mathrm{~d}-\mathrm{f}$, respectively. 


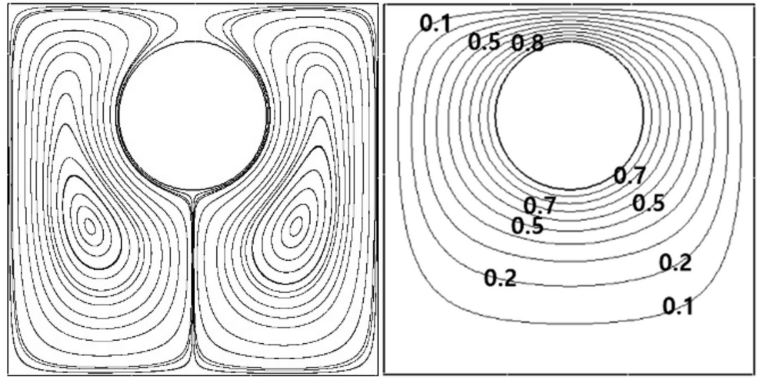

(a) Mode II
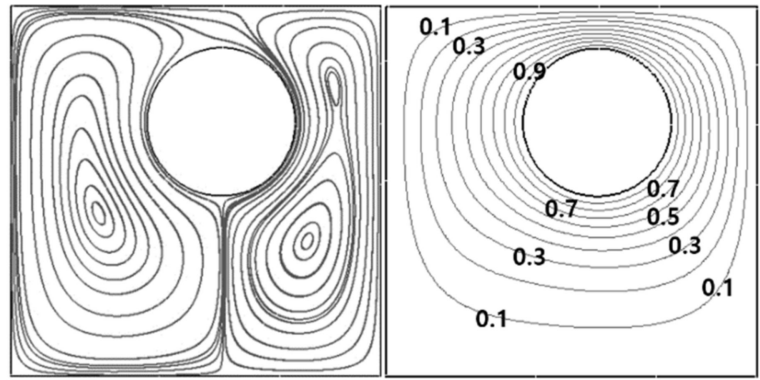

(b) Mode III
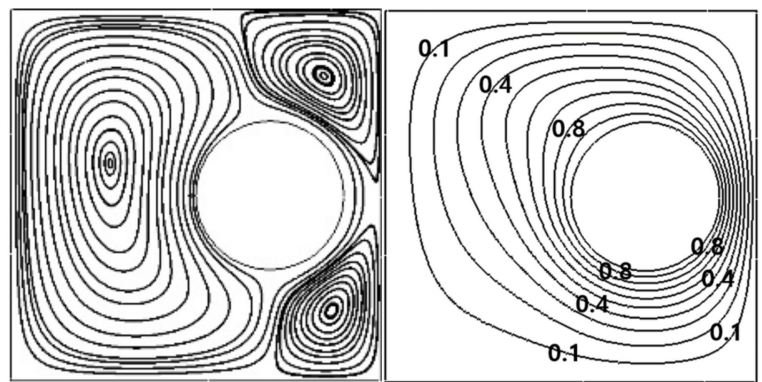

(c) Mode IV

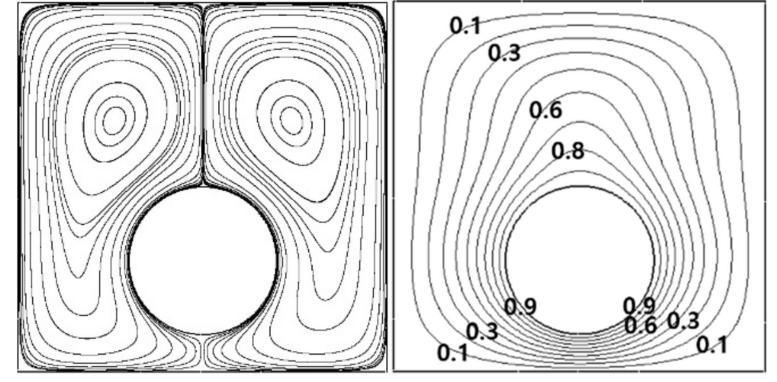

(d) Mode II

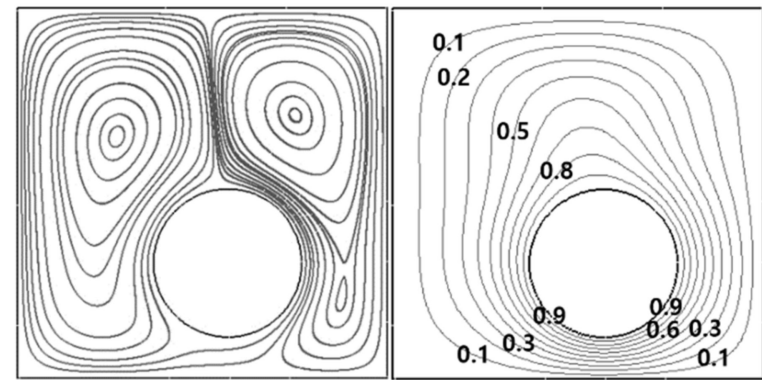

(e) Mode III
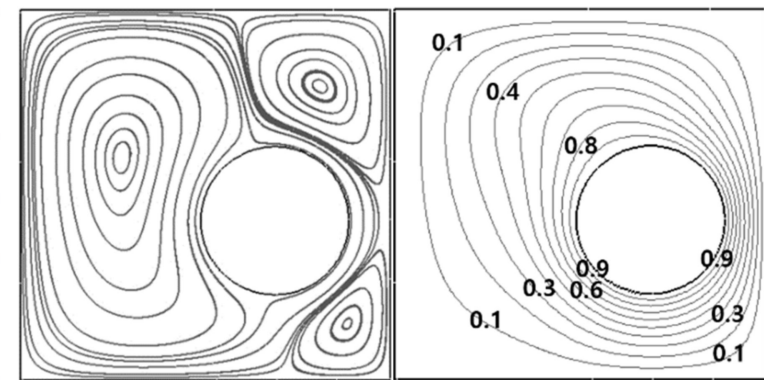

(f) Mode IV

Figure 12. Streamlines (left column) and isotherms (right column) at (a) $\theta=90^{\circ}$, (b) $\theta=70^{\circ}$, (c) $\theta=0^{\circ}$ (d) $\theta=-90^{\circ}$, (e) $\theta=-70^{\circ}$ and (f) $\theta=-20^{\circ}$ at a fixed $r=0.2 L$ for $R a=10^{4}$.

At $r=0.25 \mathrm{~L}$, when the inner cylinder rotates, the bifurcation from Mode II to Mode III occurs later than that at $r=0.2 \mathrm{~L}$, resulting in that the size of Mode II at $r=0.25 \mathrm{~L}$ is wider than that at $r=0.2 L$, as observed in Figure 9 for the Map. In comparison with $R a=10^{3}$, the region for Mode IV enlarges when $R a=10^{4}$.

\subsection{3. $R a=10^{5}$}

When $R a=10^{5}$, the Mode I with TPE_TIV, existed at $R a=10^{4}$ and $10^{4}$, disappears in the map of the flow structures, as shown in Figure 13. The new three modes of V, VI and VII appear. The Mode I is divided into about the four modes of II, III, V, VI and VII.

The center of the enclosure is occupied by Mode II, since a stronger buoyancy makes merging the lower inner vortex into the upper vortex of Mode I which is existed at $R a=10^{3}$ and $10^{4}$. Therefore, the centers of the overall rotating eddies locate at the upper half of the enclosure and the upward thermal plume is originated from the internal cylinder in Figure 14a.

The radial eccentricity by the cylinder downward movement along the vertical centerline from $\left(r=0, \theta=-90^{\circ}\right)$, the Mode II keep maintains, as shown Figure $14 \mathrm{~b}$ for $r=0.25 \mathrm{~L}$, respectively. As the cylinder moves downward from the enclosure center, the circulation is getting larger in the upper half of the enclosure and the upward thermal plume is more elongated, since wider area between the bottom wall and the cylinder secures a stronger convection in the upper half of the enclosure. 


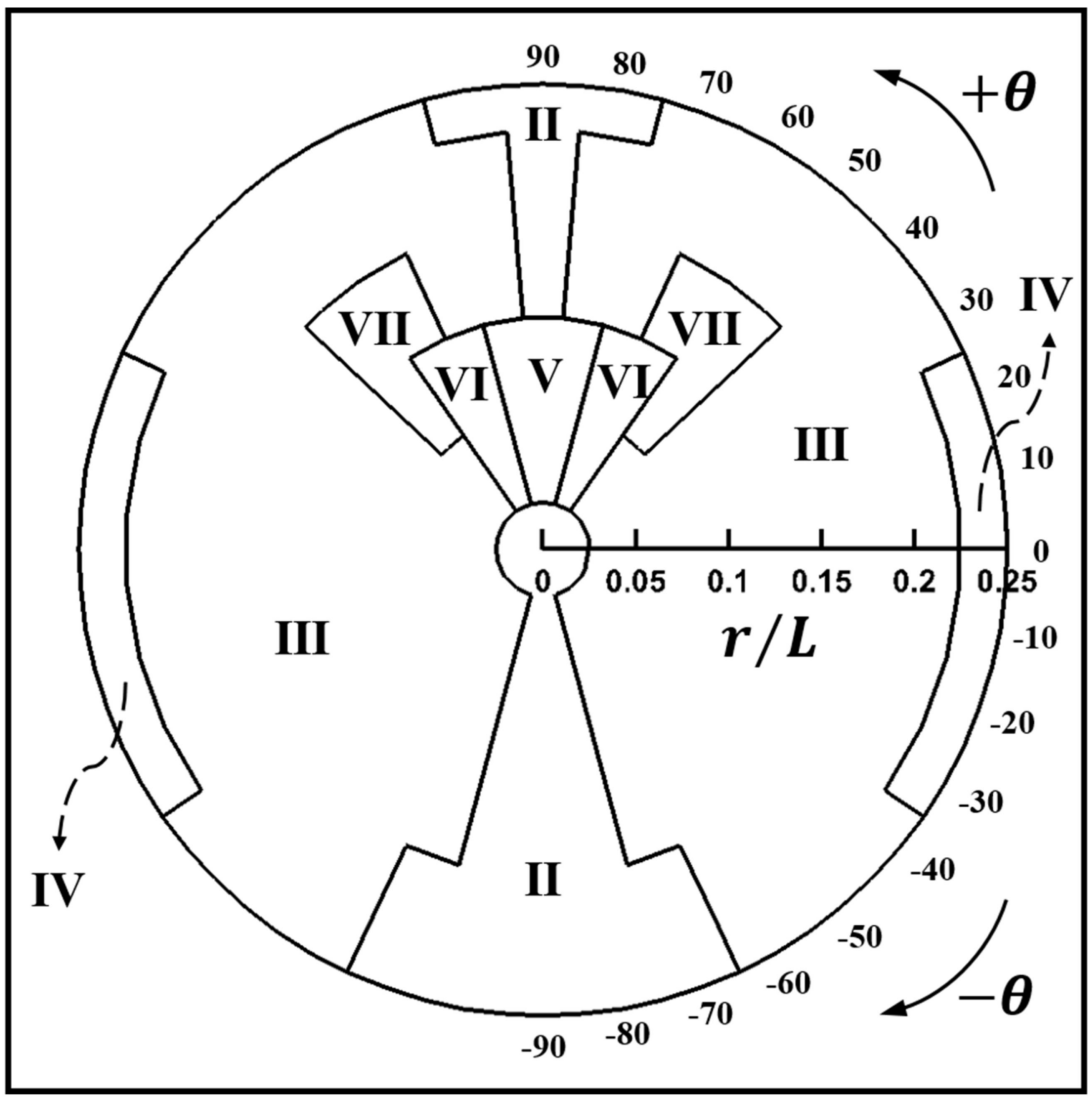

Figure 13. Map of the flow structure for $R a=10^{5}$.

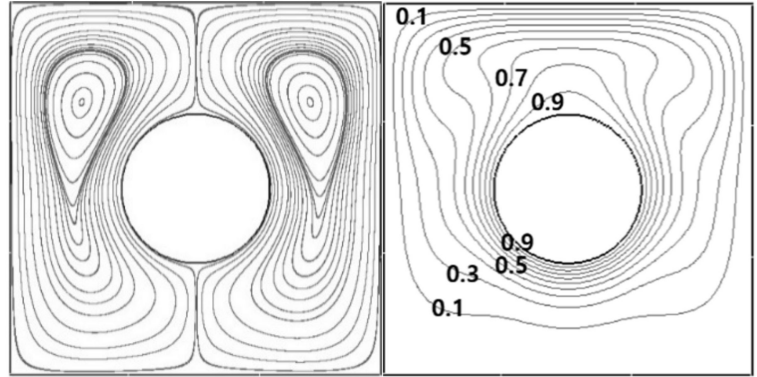

(a) Mode II

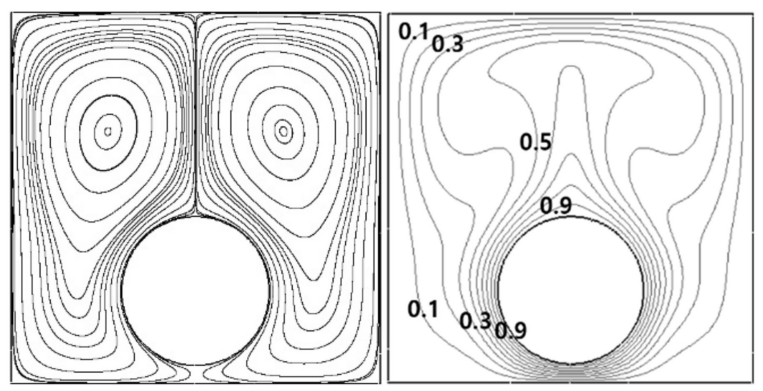

(b) Mode II

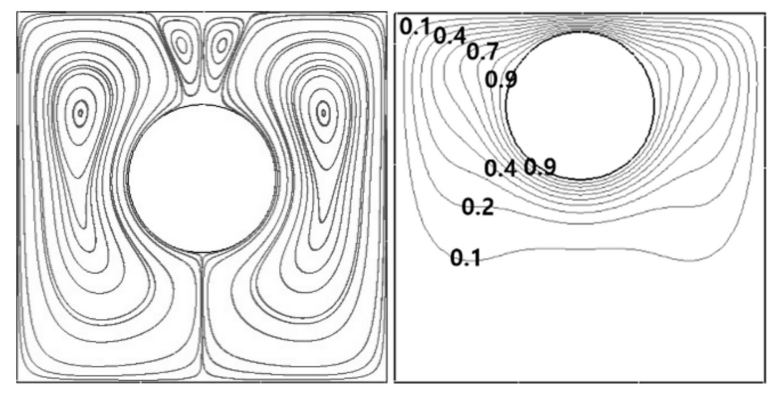

(c) Mode V

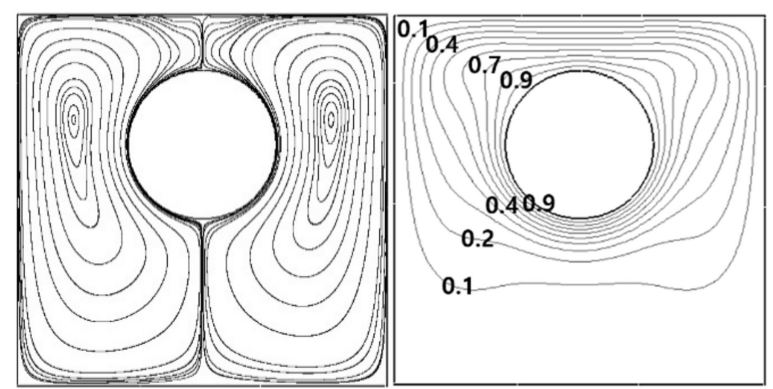

(d) Mode II

Figure 14. Streamlines (left column) and isotherms (right column) at (a) $\left(r=0, \theta=0^{\circ}\right),(\mathbf{b})\left(r=0.25 L, \theta=-90^{\circ}\right),(\mathbf{c})(r=0.05 L$, $\left.\theta=90^{\circ}\right)$ and $(\mathbf{d})\left(r=0.15 \mathrm{~L}, \theta=90^{\circ}\right)$ for $R a=10^{5}$.

The upward movement of the cylinder from $\left(r=0, \theta=90^{\circ}\right)$ contributes to the presence of the new Mode V in the Map of the flow structure in Figure 13. When the cylinder moves upward from the enclosure center, the mode changes from Mode II to Mode V and 
then recovers again Mode II, which is clearly identified in Figure 14c,d for $r=0.05 L$ and $r=0.15 L$, respectively.

In the range of $0.05 L \leq r \leq 0.1 L$ corresponding to Mode $\mathrm{V}$ in the map, a fair of secondary vortices appears in the cylinder upper surface. Therefore, Mode V presents two overall rotating primary eddies and secondary two vortices (TPE_STV). The corresponding isothermal lines exhibit that one upward plume in Mode II is divided into two plumes from the cylinder and one downward plume from the enclosure top wall, respectively, as presented in Figure 14c.

As the cylinder moves further upward from $r=0.1 \mathrm{~L}$ to $r=0.25 \mathrm{~L}$, the area between the enclosure top wall and cylinder is not enough to activate the secondary two vortices in this $R a=10^{5}$. In other words, the buoyance force is not enough to overcome the conduction and to keep the convection to maintain the secondary two vortices in this narrow space at this $R a=10^{5}$. Thus, the secondary two vortices disappear, as shown in Figure $14 \mathrm{~d}$ for $r=0.15 \mathrm{~L}$. Mainly, the isotherms are linearly distributed in this narrow region between the enclosure top wall and cylinder. As a result, Mode V changes into Mode II.

The circumferential eccentricity by the clockwise rotation of the cylinder from $(r=0.05 \mathrm{~L}$, $\left.\theta=90^{\circ}\right)$ to the horizontal center line $\left(r=0.05 \mathrm{~L}, \theta=0^{\circ}\right)$ leads to two bifurcations from Mode $\mathrm{V}$ to Mode VI and from Mode VI to Mode III, as shown in Figure 13. When $\theta=70^{\circ}$ at $r=0.05 \mathrm{~L}$, the upper secondary vortices still appear, but these secondary vortices become asymmetric, since the left upper secondary vortex is squeezed and smaller by the augmentation of the left primary eddy due to the enlargement of the left upper region by the clockwise rotation of the cylinder. Particularly, the right primary eddy contains additional tiny lower inner vortex by the compression of the clockwise rotation of the cylinder, as presented in Figure 15a.
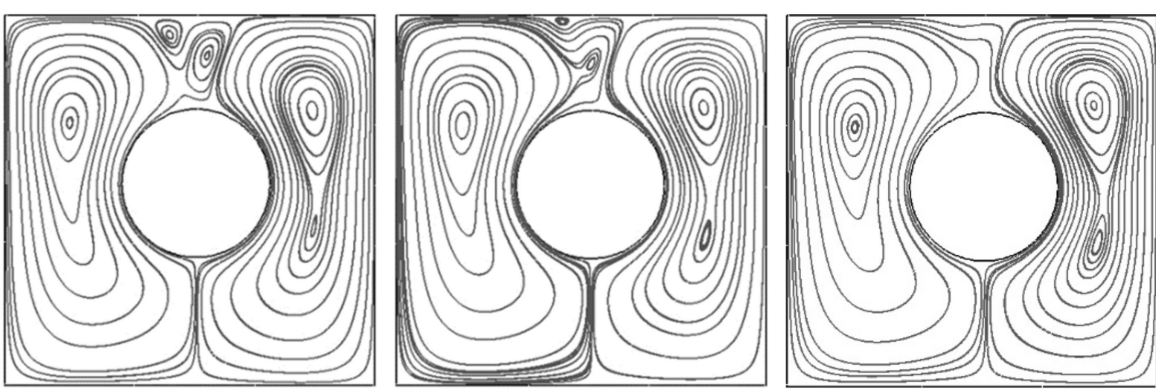

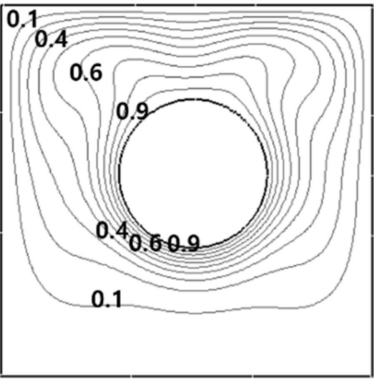

(a) Mode VI

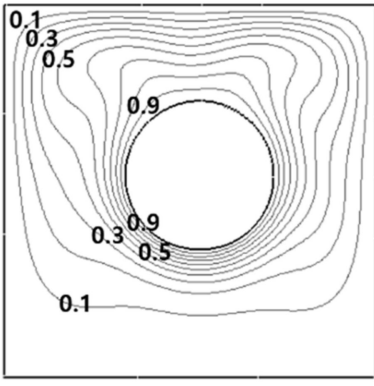

(b) Mode VI

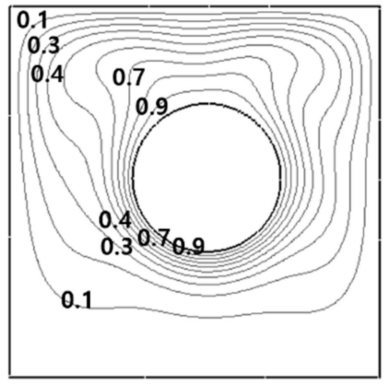

(c) Mode III

Figure 15. Streamlines (upper row) and isotherms (lower row) at (a) $\theta=70^{\circ}$, (b) $\theta=60^{\circ}$ and (c) $\theta=50^{\circ}$ at a fixed $r=0.05 \mathrm{~L}$ for $R a=10^{5}$.

As a result, Mode VI forms secondary two vortices and two overall rotating primary eddies which have two inner vortices in a large circulation in the direction of the inner cylinder rotation and one inner vortex in the opposite primary circulation. Finally, Mode VI is named as TPE_TOIV_STV. When the inner cylinder clockwise rotates from $\theta=70^{\circ}$ to $\theta=50^{\circ}$ at $r=0.05 \mathrm{~L}$, the upper secondary vortices becomes smaller but the lower inner vortex in the right primary eddy becomes bigger at $\theta=60^{\circ}$ in Figure $15 \mathrm{~b}$, and then the 
upper secondary vortices disappears at $\theta=50^{\circ}$ in Figure $15 \mathrm{c}$. Therefore, at $\theta=50^{\circ}$, the bifurcation from Mode VI to Mode III occurs. The Mode III maintains up to $\theta=0^{\circ}$.

At $r=0.1 L$, the circumferential eccentricity by the cylinder clockwise rotating from $\theta=90^{\circ}$ to the horizontal center line of $\theta=0^{\circ}$ leads to three bifurcations from Mode $\mathrm{V}$ to Mode VI, from Mode VI to Mode VII, and from Mode VII to Mode III, as shown in Figure 13 for the Map.

At $r=0.1 L$, when the inner cylinder clockwise rotates from $\theta=90^{\circ}$ to $\theta=60^{\circ}$, first bifurcation from Mode V to Mode VI occurs, as shown in Figure 16a. When $\theta=45^{\circ}$, the left upper secondary vortex disappears and simultaneously the right upper secondary vortex becomes smaller, as shown in Figure 16b. Thus, new flow structure forms in Mode VII where one secondary upper vortex in company with TPE_TOIV. The Mode VII is defined as TPE_TOIV_SOV. When the inner cylinder reaches to $\theta=40^{\circ}$, the right upper secondary vortex also disappears, as shown in Figure 16c, resulting in Mode III which maintains to $\theta=0^{\circ}$.
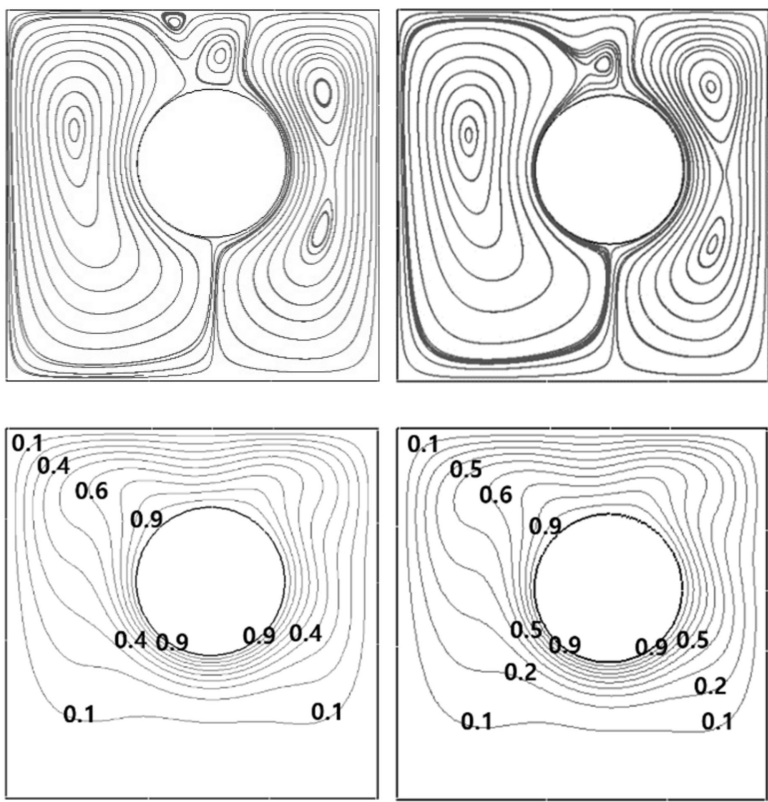

(a) Mode VI

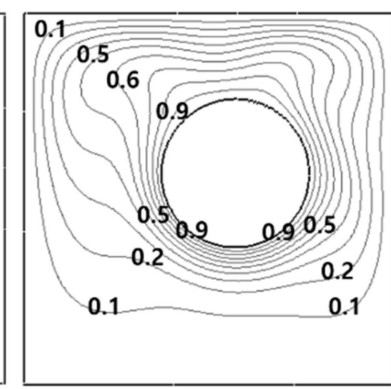

(b) Mode VII
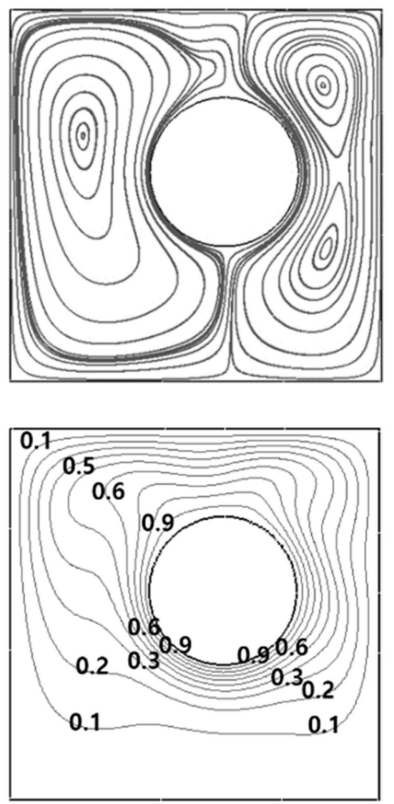

(c) Mode III

Figure 16. Streamlines (upper row) and isotherms (lower row) at (a) $\theta=60^{\circ}$, (b) $\theta=45^{\circ}$ and (c) $\theta=40^{\circ}$ at a fixed $r=0.1 L$ for $R a=10^{5}$.

At $r=0.15 \mathrm{~L}$, the clockwise rotation of the inner cylinder from $\theta=90^{\circ}$ to $\theta=0^{\circ}$ derives three bifurcations from Mode II to Mode III, from Mode III to Mode VII, and from Mode VII to Mode III, as shown in Figure 14 for the Map. At this radial location, Mode II at $\theta=90^{\circ}$ changes into Mode III at $\theta=80^{\circ}$, and the lower inner vortex of the primary eddy in the rotational direction becomes bigger at $\theta=70^{\circ}$, as shown in Figure 17a for $\theta=80^{\circ}$.

When $\theta=60^{\circ}$ at $r=0.15 \mathrm{~L}$, the secondary tiny vortex appears on the cylinder upper surface and it exists up to $\theta=45^{\circ}$ under the main flow structure of Mode III, as shown in Figure $17 \mathrm{~b}$ for $\theta=60^{\circ}$. The primary circulation in the opposite direction to the rotation of the cylinder strongly turns around the inner cylinder surface in the opposite side circulation, as the inner cylinder rotates clockwise from $\theta=90^{\circ}$.

When $\theta=40^{\circ}$ at $r=0.15 \mathrm{~L}$, the secondary tiny vortex disappears by the augmentation in size and strength of the upper inner vortex of the primary eddy in the opposite direction to the rotation of the inner cylinder, resulting in Mode III which is preserved up to $\theta=0^{\circ}$, as shown in Figure $17 \mathrm{c}$ at $\theta=40^{\circ}$. Therefore, at $r=0.15 \mathrm{~L}$, the third bifurcation from Mode VII to Mode III occurs at $\theta=40^{\circ}$. 

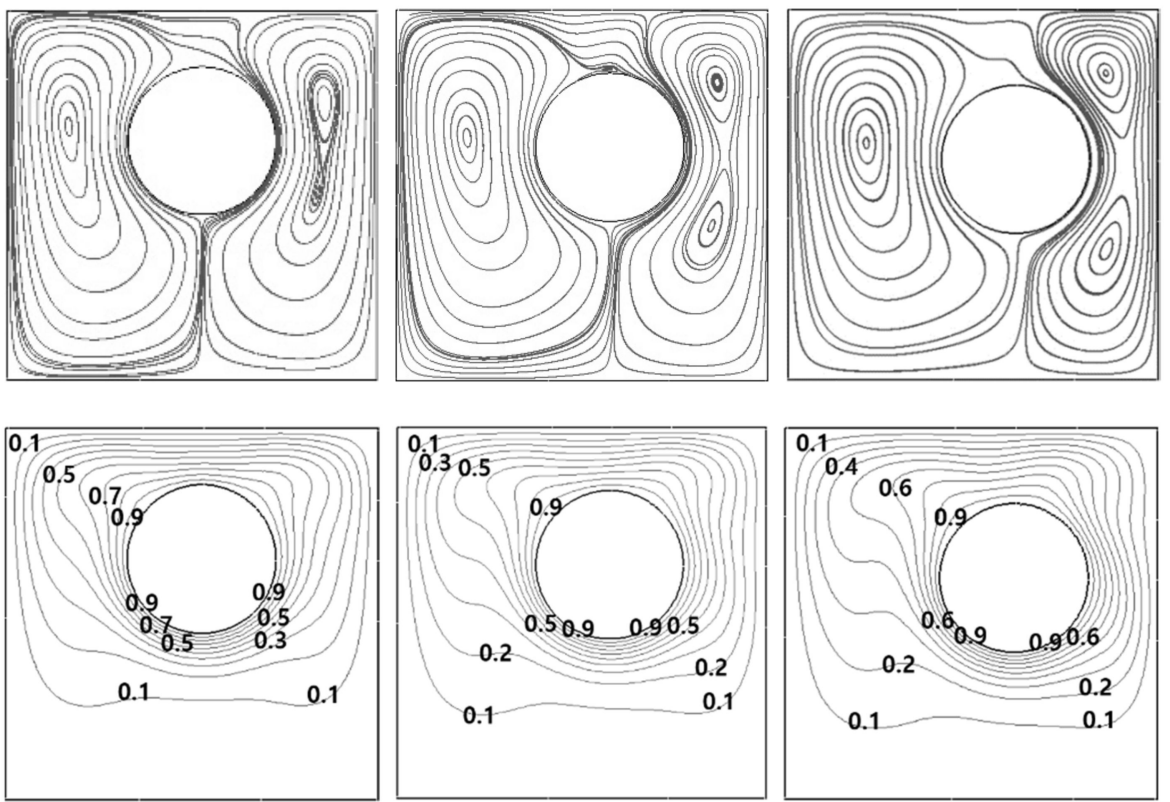

(b) Mode VII

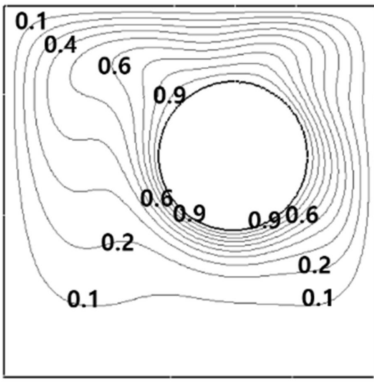

(c) Mode III (a) Mode III

Figure 17. Streamlines (upper row) and isotherms (lower row) at (a) $\theta=80^{\circ}$ (b) $\theta=60^{\circ}$ and (c) $\theta=40^{\circ}$ at a fixed $r=0.15 \mathrm{~L}$ for $\mathrm{R} a=10^{5}$.

At $r=0.2 L$, when the inner cylinder rotates clockwise from $\theta=90^{\circ}$ to $\theta=0^{\circ}$ and counterclockwise from $\theta=-90^{\circ}$ to $\theta=0^{\circ}$, single bifurcation from Mode II to Mode III occurs at $\theta=80^{\circ}$ and $\theta=-60^{\circ}$, respectively, as shown in Figure 13 for the Map.

At $r=0.25 \mathrm{~L}$, when the inner cylinder rotates clockwise from $\theta=90^{\circ}$ to $\theta=0^{\circ}$, two bifurcations occur, as shown in Figure 13. First bifurcation from Mode II to Mode III occurs at $\theta=70^{\circ}$, as shown in Figure 18 a for $\theta=70^{\circ}$. The second bifurcation from Mode III to Mode IV occurs at $\theta=20^{\circ}$ and Mode IV preserves up to $\theta=0^{\circ}$, as shown in Figure $18 \mathrm{~b}$ for $\theta=20^{\circ}$.

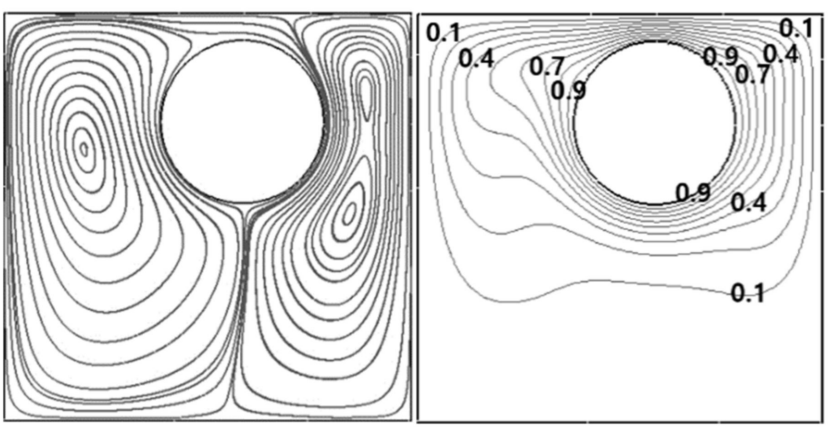

(a) Mode III

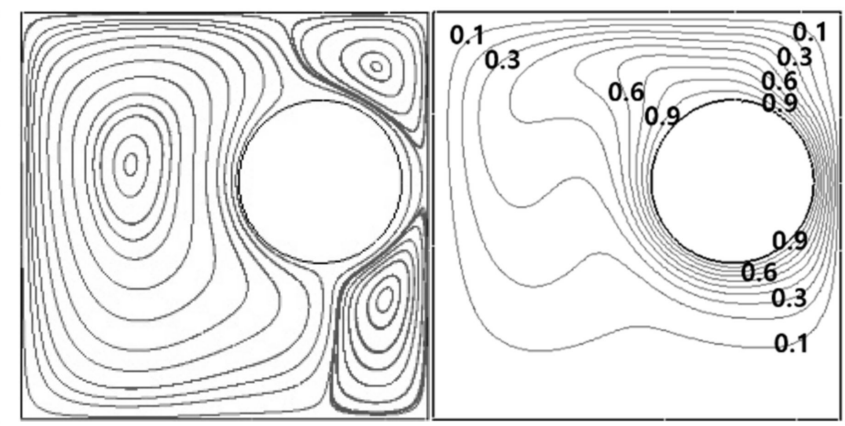

(b) Mode IV

Figure 18. Streamlines (left column) and isotherms (right column) at (a) $\theta=70^{\circ}$ and (b) $\theta=20^{\circ}$ at a fixed $r=0.25 \mathrm{~L}$ for $R a=10^{5}$.

At $r=0.25 \mathrm{~L}$, the counter-clockwise rotation from $\theta=-90^{\circ}$ to $\theta=0^{\circ}$ provides two bifurcations, as shown in Figure 13. First bifurcation from Mode II to Mode III occurs at $\theta=-60^{\circ}$, as shown in Figure 19a. The second bifurcation from Mode III to Mode IV occurs at $\theta=-30^{\circ}$, as shown in Figure $19 \mathrm{~b}$. 


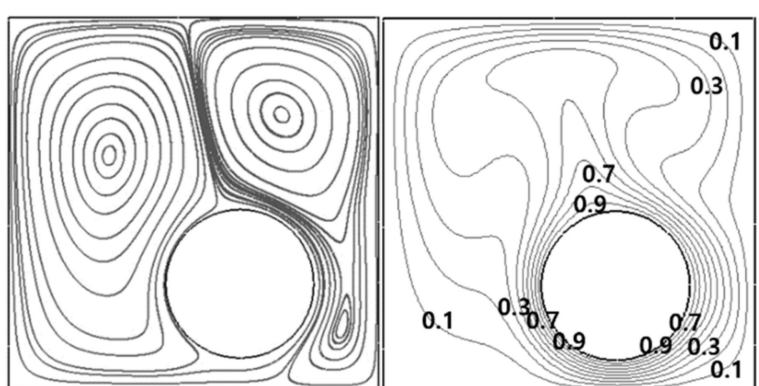

(a) Mode III

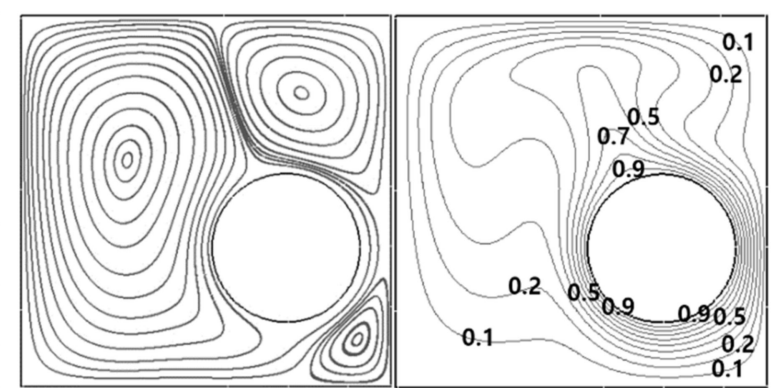

(b) Mode IV

Figure 19. Streamlines (left column) and isotherms (right column) at (a) $\theta=-60^{\circ}$ and (b) $\theta=-30^{\circ}$ at a fixed $r=0.25 \mathrm{~L}$ for $R a=10^{5}$.

The circumferential eccentricity by the counter-clockwise rotation from $\theta=-90^{\circ}$ to $\theta=0^{\circ}$ in the lower half leads to almost the same distribution of isotherms, regardless of the radial position. At $\theta=-90^{\circ}$, the isotherms form upward and large plume appears on the cylinder, which forms sharper thermal gradient near the enclosure top. The dense isotherms form over the lower surface of the cylinder due to the increased buoyance with increasing Rayleigh number. The stagnant area under the cylinder becomes wider with augmenting $r$, resulting in very coarse isotherms. When the inner cylinder rotates counterclockwise from $\theta=-90^{\circ}$ to $\theta=0^{\circ}$, the upward thermal plume inclines to the cylinder rotational direction.

\subsection{Nusselt Number}

The distributions of the surface-averaged Nusselt numbers $(\langle\overline{N u}\rangle)$ for each walls of the enclosure are presented as the contours in the $(r, \theta)$ plane in the $(r, \theta)$ plane embedded in the enclosure in order to match with the map of the flow structures according to the positions of the cylinder center, as presented in Figure 20.

For $R a=10^{3}$, the gap between the surface of the enclosure wall and the cylinder is predominant to the change of $\langle\overline{N u}\rangle$, as shown in Figure 20a-c for the top, bottom, right walls of the enclosure, respectively. The $\langle\overline{N u}\rangle$ for the bottom and top walls are dependent on nearly the vertical direction, as shown in Figure 20a,b, respectively, due to the strong conduction at low $R a=10^{3}$. Because the upward movement of the cylinder forms denser and coarser isotherms near the top and bottom walls, respectively, the larger and smaller values of $\langle\overline{N u}\rangle$ for the top and bottom walls appear at $\theta=90^{\circ}$ and $\theta=-90^{\circ}$ for $r=0.25 \mathrm{~L}$. The cylinder downward movement derives the opposite patterns of $\langle\overline{N u}\rangle$ for the bottom and top walls to the upward movement.

At this low $R a=10^{3}$, the right wall exhibits the symmetric distribution of $\langle\overline{N u}\rangle$ about the center line of $x=0$, observed in Figure 20c. The values of $\langle\overline{N u}\rangle$ for the right wall is dependent on nearly the horizontal direction. Therefore, the distribution of $\langle\overline{N u}\rangle$ is composed of almost the vertical lines. However, the careful observation identifies the very weak curvature of the contours which means the existence of the weak convection at low $R a=10^{3}$. The magnitude of $\langle\overline{N u}\rangle$ for the right wall is governed by the distance between the enclosure wall and the cylinder surface, like that for the bottom and top walls.

For $R a=10^{4}$, the distributions of $\langle\overline{N u}\rangle$ for the enclosure walls are almost the same as $R a=10^{3}$, which is consistent with the similar maps for the thermal and flow structures for $R a=10^{4}$ and $R a=10^{3}$. Therefore, the $\langle\overline{N u}\rangle$ for the bottom and top walls depend on mainly the vertical distance and form the horizontal lines based contours, as presented in Figure 20d,e. The $\langle\overline{N u}\rangle$ for the right wall is dependent on nearly the horizontal direction. Thus, the distribution of $\langle\overline{N u}\rangle$ is composed of almost the vertical lines, as shown in Figure 20f. However, the increase of the convection with augmenting $R a$ from $10^{3}$ and $R a=10^{4}$ contributes the contours of $\langle\overline{N u}\rangle$ to be more curvature. 


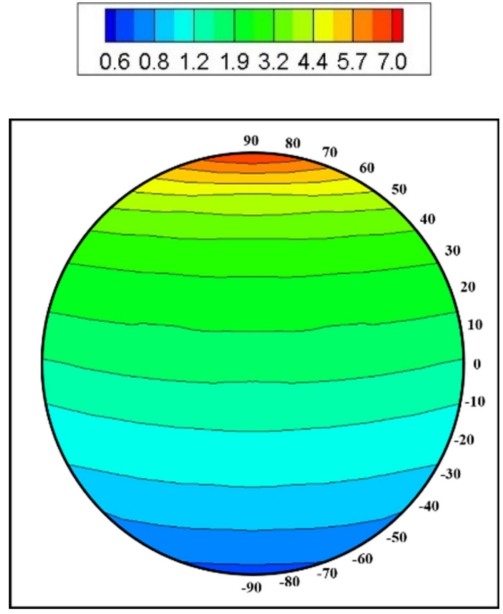

(a)

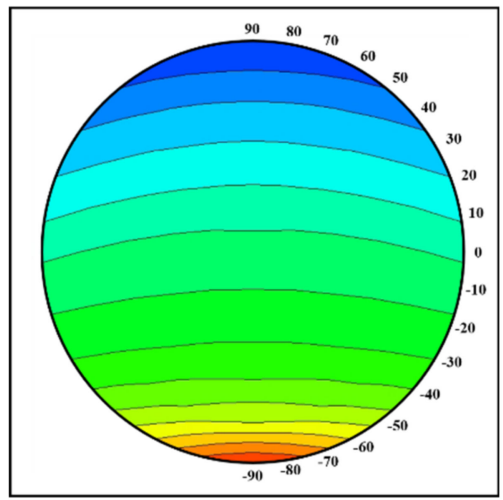

(b)

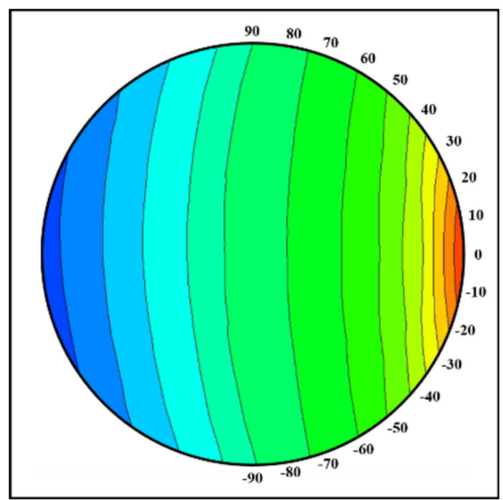

(c)
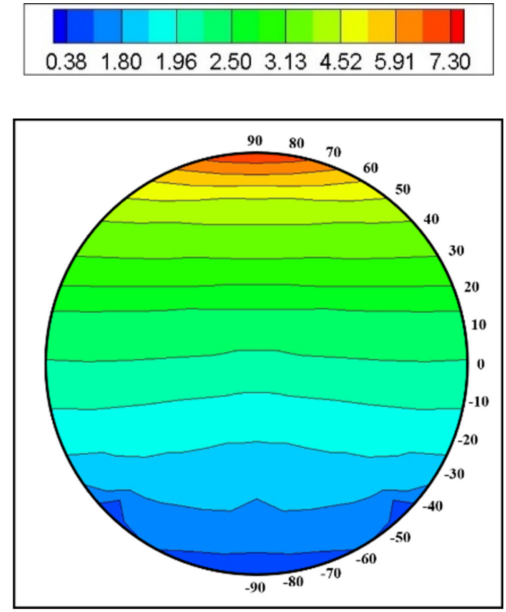

(d)

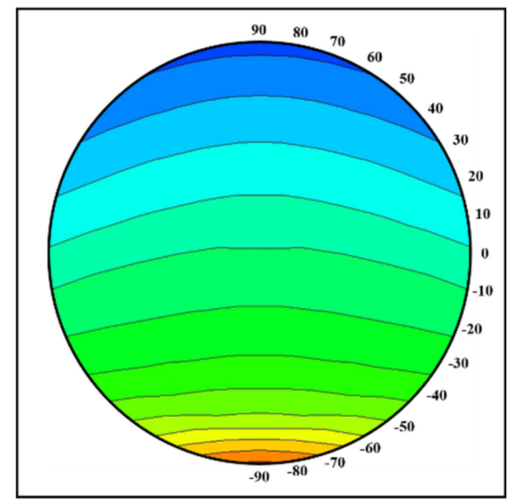

(e)

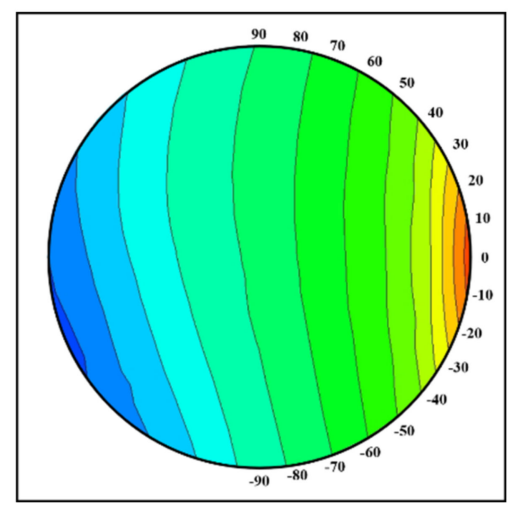

(f)
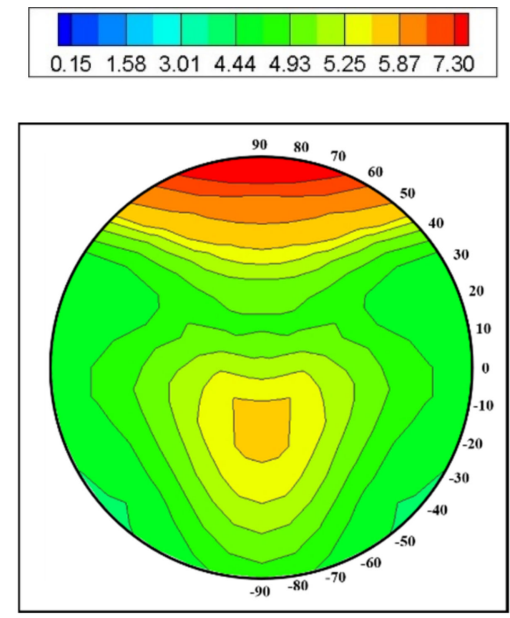

(g)

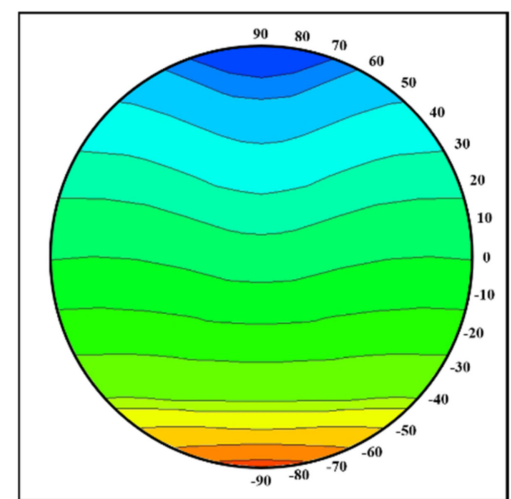

(h)

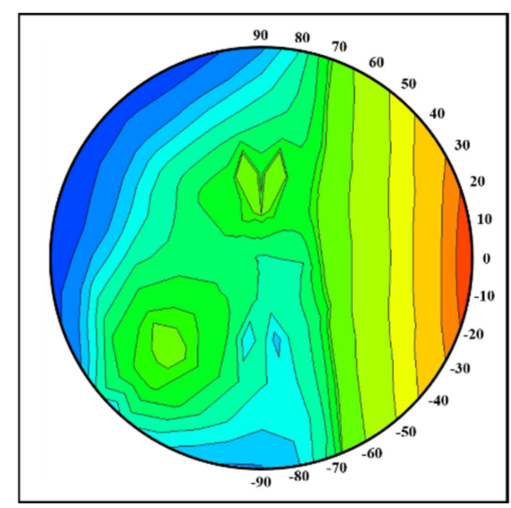

(i)

Figure 20. Contours of the top [1st row, $(\mathbf{a}, \mathbf{d}, \mathbf{g})]$, bottom [2nd row, $(\mathbf{b}, \mathbf{e}, \mathbf{h})]$ and right $[3$ rd row, $(\mathbf{c}, \mathbf{f}, \mathbf{i})]$ surface-averaged Nusselt numbers for $R a=10^{3}$ (1st column), $10^{4}$ ( 2 nd column) and $10^{5}$ (3rd column).

As $R a$ becomes large to $R a=10^{5}$, the distributions of $\langle\overline{N u}\rangle$ for the walls of the enclosure considerably change in comparison with those for lower $R a=10^{3}$ and $R a=10^{4}$, which can be clarified in Figure 20g-i, a-c and d-f for $R a=10^{5}, R a=10^{3}$ and $R a=10^{4}$, respectively.

As discussed in the thermal and flow structures, when $R a=10^{5}$, the large upward thermal plume form the cylinder upper surface characterizes these fields due to the increase of the buoyance. Therefore, the magnitudes of $\langle\overline{N u}\rangle$ for the top wall becomes much bigger than those for lower $R a=10^{3}$ and $R a=10^{4}$.

As the cylinder moves upward along the centerline from $\left(r=0.25 \mathrm{~L}, \theta=-90^{\circ}\right)$ which is the nearest cylinder position to the bottom wall, the $\langle\overline{N u}\rangle$ for the top wall increases, 
decreases and increases again. The increase of $\langle\overline{N u}\rangle$ according the upward movement of the cylinder from $\left(r=0.25 \mathrm{~L}, \theta=-90^{\circ}\right)$ is attributed to the combined effects of the augmentation of the conduction due to the reduction gap between the cylinder and the top wall and a stronger convection generating the large upward thermal plume. As the cylinder keeps moving upward, the value of $\langle\overline{N u}\rangle$ decreases by the slightly weaken convection due to a smaller area between the cylinder and the top wall. Also, the downward plume from the top wall of the enclosure, corresponding to the secondary vortices over the upper surface of the inner cylinder, contributes to the decrease of $\langle\overline{N u}\rangle$ for the top wall. Finally, as the internal cylinder continuously moves upward, the $\langle\overline{N u}\rangle$ increases and reaches to the maximum.

When the cylinder becomes far away, the upward thermal plume inclines and forms coarse isotherms near the top wall. Therefore, this inclined thermal plume contributes the value of $\langle\overline{N u}\rangle$ for the top wall to the reduction. The small $\langle\overline{N u}\rangle$ distribute around the outer boundary of the position of the inner cylinder, as presented in Figure 20g.

As the cylinder locates in the lower half of the enclosure for $R a=10^{5}$, the distribution of $\langle\overline{N u}\rangle$ for the bottom wall is similar to that for lower $R a=10^{3}$ and $R a=10^{4}$. Namely, when inner cylinder moves upward from $\left(r=0.25 \mathrm{~L}, \theta=-90^{\circ}\right)$ to the enclosure center $(r=0$, $\left.\theta=0^{\circ}\right)$, the $\langle\overline{N u}\rangle$ decreases, as shown in Figure 20h. Otherwise, when the inner cylinder locates in the upper half for $R a=10^{5}$, the variation of $\langle\overline{N u}\rangle$ is very weak with a small value, since the thermal stratification appears in the area under the cylinder, as early observed in the isotherms.

As the cylinder locates in the right half of the enclosure for $R a=10^{5}$, the $\langle\overline{N u}\rangle$ for the right wall depends on mainly the gap between the right wall and cylinder, resulting in the same increasing pattern of $\langle\overline{N u}\rangle$ with closer to the right wall as lower $R a=10^{3}$ and $R a=10^{4}$, as observed in Figure 20i. Otherwise, as the cylinder locates in the left half of the enclosure, the coarse isotherms form the opposite corners. Hence, the small $\langle\overline{N u}\rangle_{\mathrm{s}}$ for the right wall appear as the cylinder positions near the left bound of the position of the cylinder.

Figure 21 shows the total surfaces-averaged Nusselt number of enclosure, $\overline{N u}_{e n}$, in the present ranges of the radial and circumferential locations of the inner cylinder center for different $R a$. The $R a=10^{3}$ and $10^{4}$ form about the same distribution of $\overline{N u}_{e n}$, which is almost the four-fold symmetry about $x=0$ and $y=0$, as presented in Figure 21a, $\mathrm{b}$, respectively. As discussed in the thermal and flow fields, because the gap is predominant to the heat transfer, $\overline{N u}_{e n}$ has large values at the horizontal and vertical boundaries of the cylinder position. In other words, the magnitude of $\overline{N u}_{e n}$ is mainly as a function of the radial direction at these low $R a$ of $10^{3}$ and $10^{4}$. For the validation in Figure $3, \overline{N u}_{e n}$ has a parabolic profile according to $r$, regardless of $\theta$.

As $R a$ increases to $10^{5}$, the location of the minimum of $\overline{N u}_{e n}$ moves diagonally upward from the center of the enclosure $(x=0, y=0)$ where the minimum of $\overline{N u}_{\text {en }}$ occurs at $R a=10^{3}$ and $10^{4}$ in Figure 21c. In the regions showing the minimum of $\overline{N u}_{e n}$, the inclined rising thermal plumes on the upper surface of the cylinder form the secondary vortices which accompany the down welling plume from the top wall of the enclosure. This down welling plume derives the coarse isotherms near the enclosure top wall, which contributes to forming regions containing the minimum of $\overline{N u}_{e n}$. The value of $\overline{N u}_{e n}$ near the bottom are bigger than that those near the top wall, because the stagnant region at the top wall becomes wider than that near the bottom. 


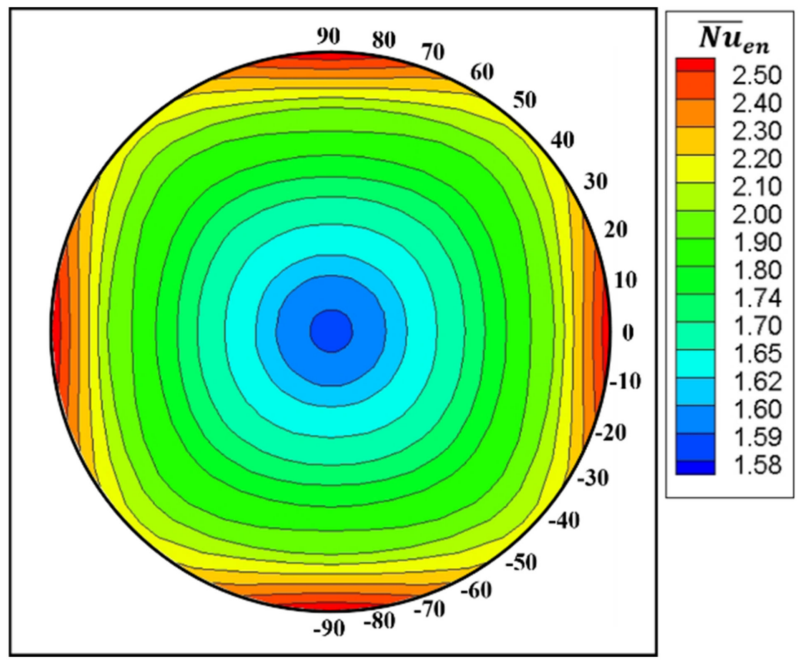

(a)

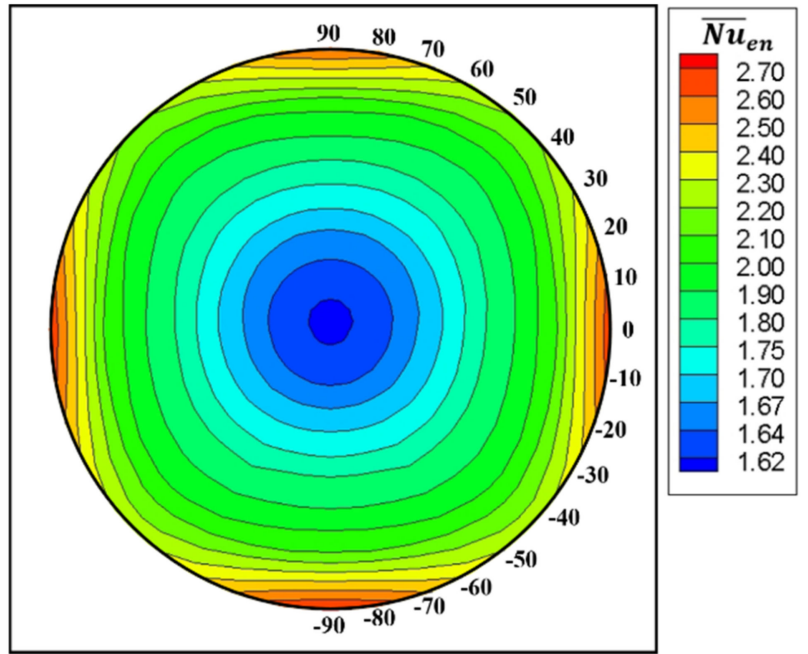

(b)

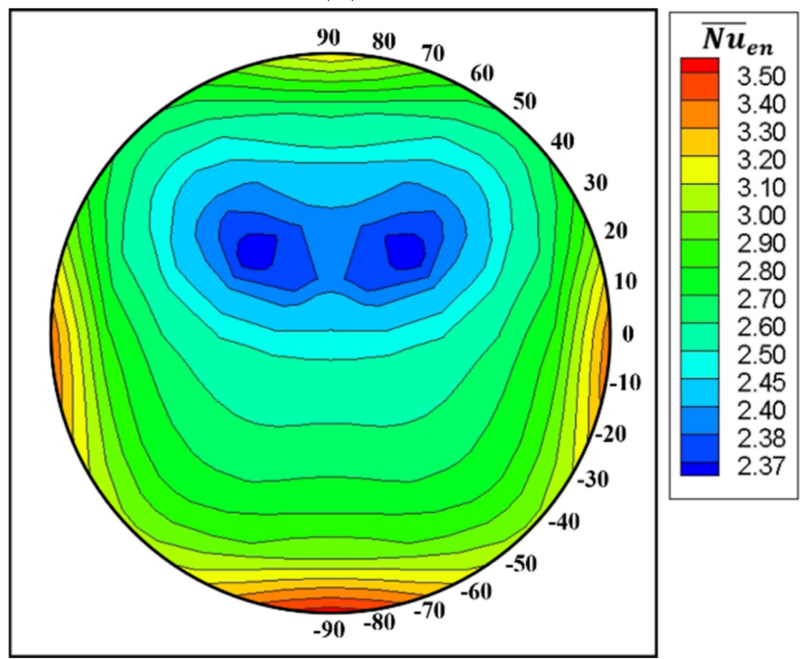

(c)

Figure 21. Total surface-averaged Nusselt number of the enclosure for (a) $R a=10^{3}$, (b) $R a=10^{4}$ and (c) $R a=10^{5}$. 


\section{Conclusions}

The present study researched the natural convection in a cold square enclosure with a hot circular cylinder. The numerical simulations are performed to solve a 2-D unsteady natural convection for $R a$ of $R a=10^{3}, 10^{4}$ and $10^{5}$ at $P r=0.7$.

This study covered the wide range of the cylinder positions to identify the eccentric effect of the inner cylinder on flow and thermal structures in the square enclosure. The present study classified the flow structures according to the cylinder center in the enclosure. The isotherms were presented to explain the bifurcation of the flow structure.

The maps for the flow structures were provided to reinforce the understanding of the eccentricity of the cylinder within the enclosure for different Rayleigh numbers. The Rayleigh numbers of $10^{3}$ and $10^{4}$ form the four modes of the flow structure. These modes are classified by mainly the large circulation and inner vortices. Therefore, the maps for these Rayleigh numbers consist of four modes.

Mode I reveals two overall rotating primary eddies and each rotating eddy has two inner vortices (TPE_TIV). When the inner cylinder gets away from the boundary of Mode I, the two inner vortices are merged. Mode II reveals two overall rotating primary eddies (TPE). When the inner cylinder gets away from the boundary of Mode II, the primary circulation in the inner cylinder rotating direction contains additional inner vortex. Therefore, Mode III reveals two overall rotating primary eddies which have two inner vortices in the primary circulation in the direction of the inner cylinder rotation and one inner vortex in the opposite primary circulation (TPE_TOIV). When the inner cylinder gets away from the boundary of Mode III, two inner eddies embedded in the primary eddy in the direction of the inner cylinder rotation are divided. Therefore, Mode IV reveals one overall rotating primary eddy and two divided vortices (OPE_TDV).

When $R a=10^{5}$, the Mode I with TPE_TIV, existed at $R a=10^{3}$ and $10^{4}$, disappears in the map of the flow structures. The new three modes of V, VI and VII appear. Mode $\mathrm{V}$ presents two overall rotating primary eddies and secondary two vortices (TPE_STV). Mode VI forms secondary two vortices and two overall rotating primary eddies which have two inner vortices in the primary circulation in the direction of the inner cylinder rotation and one inner vortex in the opposite primary circulation. Finally, Mode VI is named as TPE_TOIV_STV. Mode VII has one secondary upper vortex in company with TPE_TOIV. The Mode VII is defined as TPE_TOIV_SOV. At $R a=10^{5}$, these new modes are characterized by the top side secondary vortices.

Author Contributions: Supervision, H.-S.Y.; Writing-original draft, H.-S.Y.; Validation, Y.-J.S.; Software, Y.-J.S.; All authors have read and agreed to the published version of the manuscript.

Funding: This work was supported by the National Research Foundation of Korea (NRF) grant funded by the Korea government (MSIT) (NRF-2019R1A2C1009081).

Institutional Review Board Statement: Not applicable.

Informed Consent Statement: Not applicable.

Data Availability Statement: Not applicable.

Acknowledgments: This work was supported by the National Research Foundation of Korea (NRF) grant funded by the Korea government (MSIT) (NRF-2019R1A2C1009081).

Conflicts of Interest: The authors declare no conflict of interest.

\section{References}

1. Ghaddar, N.K. Natural convection heat transfer between a uniformly heated cylindrical element and its rectangular enclosure. Int. J. Heat Mass Transf. 1992, 35, 2327-2334. [CrossRef]

2. Lee, S.H.; Seo, Y.M.; Yoon, H.S.; Ha, M.Y. Three-dimensional natural convection around an inner circular cylinder located in a cubic enclosure with sinusoidal thermal boundary condition. Int. J. Heat Mass Transf. 2016, 101, 807-823. [CrossRef]

3. Bhowmick, D.; Randive, P.R.; Pati, S.; Agrawal, H.; Kumar, A.; Kumar, P. Natural convection heat transfer and entropy generation from a heated cylinder of different geometry in an enclosure with non-uniform temperature distribution on the walls. J. Therm. Anal. Calorim. 2020, 141, 839-857. [CrossRef] 
4. Park, J.; Kim, M.; Mun, G.S.; Park, Y.G.; Ha, M.Y. Natural convection in a square enclosure with a circular cylinder with adiabatic side walls according to bottom wall temperature variation. J. Mech. Sci. Technol. 2018, 32, 3201-3211. [CrossRef]

5. Roslan, R.; Saleh, H.; Hashim, I.; Bataineh, A.S. Natural convection in an enclosure containing a sinusoidally heated cylindrical source. Int. J. Heat Mass Transf. 2014, 70, 119-127. [CrossRef]

6. Dash, S.M.; Lee, T.S. Natural convection from inclined square cylinder using novel flexible forcing IB-LBM approach. Eng. Appl. Comput. Fluid Mech. 2014, 8, 91-103. [CrossRef]

7. Jelita, M.; Mudia, H.; Afriani, S.; Kasim, U.I.S.S. Analysis of fluid dynamics and heat transfer inside an elliptical cylinder in a square enclosure. Adv. Theor. Appl. Mech. 2017, 10, 11-20. [CrossRef]

8. Cho, H.W.; Park, Y.G.; Ha, M.Y. The natural convection in a square enclosure with two hot inner cylinders Part I: The effect of one elliptical cylinder with various aspect ratios in a vertical array. Int. J. Heat Mass Transf. 2018, 125, 815-827. [CrossRef]

9. Raman, S.K.; Prakash, K.A.; Vengadesan, S. Natural convection from a heated elliptic cylinder with a different axis ratio in a square enclosure. Numer. Heat Transf. Part A Appl. 2012, 62, 639-658. [CrossRef]

10. Souayeh, B.; Ben-Cheikh, N.; Ben-Beya, B. Numerical simulation of three-dimensional natural convection in a cubic enclosure induced by an isothermally-heated circular cylinder at different inclinations. Int. J. Therm. Sci. 2016, 110, 325-339. [CrossRef]

11. Seo, Y.M.; Doo, J.H.; Ha, M.Y. Three-dimensional flow instability of natural convection induced by variation in radius of inner circular cylinder inside cubic enclosure. Int. J. Heat Mass Transf. 2016, 95, 566-578. [CrossRef]

12. Yoon, H.S.; Yu, D.H.; Ha, M.Y.; Park, Y.G. Three-dimensional natural convection in an enclosure with a sphere at different vertical locations. Int. J. Heat Mass Transf. 2016, 53, 3143-3155. [CrossRef]

13. Lu, J.; Shi, B.; Guo, Z.; Chai, Z. Numerical study on natural convection in a square enclosure containing a rectangular heated cylinder. Front. Energy Power Eng. China 2009, 3, 373. [CrossRef]

14. Moukalled, F.; Diab, H.; Acharya, S. Laminar natural convection in a horizontal rhombic annulus. Numer. Heat Transf. Part A Appl. 1993, 24, 89-107. [CrossRef]

15. Moukalled, F.; Acharya, S. Natural convection in the annulus between concentric horizontal circular and square cylinders. J. Thermophys. Heat Trans. 1996, 10, 524-531. [CrossRef]

16. Yoon, H.S.; Jung, J.H.; Park, Y.G. Natural convection in a square enclosure with two horizontal cylinders. Numer. Heat Transf. Part A Appl. 2012, 62, 701-721. [CrossRef]

17. Yoon, H.S.; Park, Y.G.; Jung, J.H. Natural convection in a square enclosure with differentially heated two horizontal cylinders. Numer. Heat Transf. Part A Appl. 2014, 65, 302-326. [CrossRef]

18. Park, H.K.; Ha, M.Y.; Yoon, H.S.; Park, Y.G.; Son, C. A numerical study on natural convection in an inclined square enclosure with a circular cylinder. Int. J. Heat Mass Transf. 2013, 66, 295-314. [CrossRef]

19. Shu, C.; Zhu, Y.D. Efficient computation of natural convection in a concentric annulus between an outer square cylinder and an inner circular cylinder. Int. J. Numer. Methods Fluids 2002, 38, 429-445. [CrossRef]

20. Angeli, D.; Levoni, P.; Barozzi, G.S. Numerical predictions for stable buoyant regimes within a square cavity containing a heated horizontal cylinder. Int. J. Heat Mass Transf. 2008, 51, 553-565. [CrossRef]

21. Seo, Y.M.; Ha, M.Y.; Park, Y.G. The effect of four elliptical cylinders with different aspect ratios on the natural convection inside a square enclosure. Int. J. Heat Mass Transf. 2018, 122, 491-503. [CrossRef]

22. Zhang, P.; Zhang, X.; Deng, J.; Song, L. A numerical study of natural convection in an inclined square enclosure with an elliptic cylinder using variational multiscale element free Galerkin method. Int. J. Heat Mass Transf. 2016, 99, 721-737. [CrossRef]

23. Park, Y.G.; Ha, M.Y.; Choi, C.; Park, J. Natural convection in a square enclosure with two inner circular cylinders positioned at different vertical locations. Int. J. Heat Mass Transf. 2014, 77, 501-518. [CrossRef]

24. Dash, S.M.; Lee, T.S. Natural convection in a square enclosure with a square heat source at different horizontal and diagonal eccentricities. Numer. Heat Transf. Part A Appl. 2015, 68, 686-710. [CrossRef]

25. Shu, C.; Xue, H.; Zhu, Y.D. Numerical study of natural convection in an eccentric annulus between a square outer cylinder and a circular inner cylinder using DQ method. Int. J. Heat Mass Transf. 2001, 44, 3321-3333. [CrossRef]

26. Kim, B.S.; Lee, D.S.; Ha, M.Y.; Yoon, H.S. A numerical study of natural convection in a square enclosure with a circular cylinder at different vertical locations. Int. J. Heat Mass Transf. 2008, 51, 1888-1906. [CrossRef]

27. Yoon, H.S.; Ha, M.Y.; Kim, B.S.; Yu, D.H. Effect of the position of a circular cylinder in a square enclosure on natural convection at Rayleigh number of $10^{7}$. Phys. Fluids 2009, 21, 047101. [CrossRef]

28. Hussain, S.H.; Hussein, A.K. Numerical investigation of natural convection phenomena in a uniformly heated circular cylinder immersed in square enclosure filled with air at different vertical locations. Int. Commun. Heat Mass Transf. 2010, 37, 1115-1126. [CrossRef]

29. Park, Y.G.; Yoon, H.S.; Ha, M.Y. Natural convection in square enclosure with hot and cold cylinders at different vertical locations. Int. J. Heat Mass Transf. 2012, 55, 7911-7925. [CrossRef]

30. Park, Y.G.; Ha, M.Y.; Yoon, H.S. Study on natural convection in a cold square enclosure with a pair of hot horizontal cylinders positioned at different vertical locations. Int. J. Heat Mass Transf. 2013, 65, 696-712. [CrossRef]

31. Baranwal, A.K.; Chhabra, R. Effect of Prandtl number on free convection from two cylinders in a square enclosure. Heat Transf. Eng. 2016, 37, 545-556. [CrossRef]

32. Park, Y.G.; Ha, M.Y.; Park, J. Natural convection in a square enclosure with four circular cylinders positioned at different rectangular locations. Int. J. Heat Mass Transf. 2015, 81, 490-511. [CrossRef] 
33. Seo, Y.M.; Park, Y.G.; Kim, M.; Yoon, H.S.; Ha, M.Y. Two-dimensional flow instability induced by natural convection in a square enclosure with four inner cylinders. Part I: Effect of horizontal position of inner cylinders. Int. J. Heat Mass Transf. 2017, 113, 1306-1318. [CrossRef]

34. Seo, Y.M.; Mun, G.S.; Park, Y.G.; Ha, M.Y. Two-dimensional flow instability induced by natural convection in a square enclosure with four inner cylinders. Part II: Effect of various positions of inner cylinders. Int. J. Heat Mass Transf. 2017, 113, 1319-1331. [CrossRef]

35. Bararnia, H.; Soleimani, S.; Ganji, D.D. Lattice Boltzmann simulation of natural convection around a horizontal elliptic cylinder inside a square enclosure. Int. Commun. Heat Mass Transf. 2011, 38, 1436-1442. [CrossRef]

36. Lee, J.M.; Ha, M.Y.; Yoon, H.S. Natural convection in a square enclosure with a circular cylinder at different horizontal and diagonal locations. Int. J. Heat Mass Transf. 2010, 53, 5905-5919. [CrossRef]

37. Kang, D.H.; Ha, M.Y.; Yoon, H.S.; Choi, C. Bifurcation to unsteady natural convection in square enclosure with a circular cylinder at Rayleigh number of $10^{7}$. Int. J. Heat Mass Transf. 2013, 64, 926-944. [CrossRef]

38. Spizzichino, A.; Zemach, E.; Feldman, Y. Oscillatory instability of a 3D natural convection flow around a tandem of cold and hot vertically aligned cylinders placed inside a cold cubic enclosure. Int. J. Heat Mass Transf. 2019, 141, 327-345. [CrossRef]

39. Cho, H.W.; Seo, Y.M.; Mun, G.S.; Ha, M.Y.; Park, Y.G. The effect of instability flow for two-dimensional natural convection in a square enclosure with different arrays of two inner cylinders. Int. J. Heat Mass Transf. 2017, 114, 307-317. [CrossRef]

40. Doo, J.H.; Mun, G.S.; Ha, M.Y.; Seong, S.Y. Thermo-dynamic irreversibility induced by natural convection in square enclosure with inner cylinder. Part-II: Effect of vertical position of inner cylinder. Int. J. Heat Mass Transf. 2016, 97, 1120-1139. [CrossRef]

41. Liao, C.C.; Lin, C.A. Transitions of natural convection flows in a square enclosure with a heated circular cylinder. Appl. Therm. Eng. 2014, 72, 41-47. [CrossRef]

42. Pandey, S.; Park, Y.G.; Ha, M.Y. An exhaustive review of studies on natural convection in enclosures with and without internal bodies of various shapes. Int. J. Heat Mass Transf. 2019, 138, 762-795. [CrossRef]

43. Aghaei, A.; Sheikhzadeh, G.A.; Goodarzi, M.; Hasani, H.; Damirchi, H.; Afrand, M. Effect of horizontal and vertical elliptic baffles inside an enclosure on the mixed convection of a MWCNTs-water nanofluid and its entropy generation. Eur. Phys. J. Plus 2018, 133, 486. [CrossRef]

44. Goodarzi, M.; D'Orazio, A.; Keshavarzi, A.; Mousavi, S.; Karimipour, A. Develop the nano scale method of lattice Boltzmann to predict the fluid flow and heat transfer of air in the inclined lid driven cavity with a large heat source inside, Two case studies: Pure natural convection \& mixed convection. Phys. A Stat. Mech. Appl. 2018, 509, 210-233.

45. Pordanjani, A.H.; Aghakhani, S.; Karimipour, A.; Afrand, M.; Goodarzi, M. Investigation of free convection heat transfer and entropy generation of nanofluid flow inside a cavity affected by magnetic field and thermal radiation. J. Therm. Anal. Calorim. 2019, 137, 997-1019. [CrossRef]

46. Yousefzadeh, S.; Rajabi, H.; Ghajari, N.; Sarafraz, M.M.; Akbari, O.A.; Goodarzi, M. Numerical investigation of mixed convection heat transfer behavior of nanofluid in a cavity with different heat transfer areas. J. Therm. Anal. Calorim. 2019, 140, $2779-2803$. [CrossRef]

47. Rozati, S.A.; Montazerifar, F.; Ali Akbari, O.; Hoseinzadeh, S.; Nikkhah, V.; Marzban, A.; Abdolvand, H.; Goodarzi, M. Natural convection heat transfer of water/Ag nanofluid inside an elliptical enclosure with different attack angles. Math. Methods Appl. Sci. 2020, 1-18. [CrossRef]

48. Kim, J.; Moin, P. Application of a fractional-step method to incompressible Navier-Stokes equations. J. Comput. Phys. 1985, 59, 308-323. [CrossRef]

49. Zang, Y.; Street, R.L.; Koseff, J.R. A non-staggered grid, fractional step method for time-dependent incompressible Navier-Stokes equations in curvilinear coordinates. J. Comput. Phys. 1994, 114, 18-33. [CrossRef] 